\title{
Intramembrane protease SPP defines a cholesterol-regulated switch of the mevalonate pathway
}

Ronny Heidasch ${ }^{1,7}$, Dönem Avci ${ }^{1,2,7, \star}$, Christian Lüchtenborg ${ }^{3}$, Dipali Kale ${ }^{3}$, Hester Beard ${ }^{4}$, Torben Mentrup ${ }^{5}$, Marta Barniol-Xicota ${ }^{4}$, Bernd Schröder ${ }^{5}$, Steven Verhelst ${ }^{4,6}$, Britta Brügger $^{3}$, and Marius K. Lemberg ${ }^{1,2,8, *}$

${ }^{1}$ Center for Molecular Biology of Heidelberg University (ZMBH), DKFZ-ZMBH Alliance, 69120 Heidelberg, Germany.

${ }^{2}$ Center for Biochemistry, Medical Faculty, University of Cologne, 50931 Cologne, Germany. ${ }^{3}$ Biochemistry Center of Heidelberg University (BZH), 69120 Heidelberg, Germany. ${ }^{4}$ Department of Cellular and Molecular Medicine, University of Leuven, 3000 Leuven, Belgium.

${ }^{5}$ Institute of Physiological Chemistry, TU Dresden, 01307 Dresden, Germany.

${ }^{6}$ Leibniz Institute for Analytical Sciences ISAS, 44227 Dortmund, Germany

${ }^{7}$ These authors contributed equally to this work.

${ }^{8}$ Lead Contact.

*Correspondence: d.avci@uni-koeln.de and m.lemberg@uni-koeln.de

\begin{abstract}
Intramembrane proteolysis regulates important processes such as signaling and transcriptional and posttranslational abundance control of proteins with key functions in metabolic pathways. This includes transcriptional control of mevalonate pathway genes, thereby ensuring balanced biosynthesis of cholesterol and other isoprenoids. Our work shows that, at high cholesterol levels, signal peptide peptidase (SPP) cleaves squalene synthase (SQS), an enzyme that defines the branching point for allocation of isoprenoids to the sterol and non-sterol arms of the mevalonate pathway. This intramembrane cleavage
\end{abstract}


releases SQS from the membrane and targets it for proteasomal degradation. Regulation of this mechanism is achieved by the E3 ubiquitin ligase TRC8 that, in addition to ubiquitinating SQS in response to cholesterol levels, acts as an allosteric activator of SPP-catalyzed intramembrane cleavage of SQS and other substrates. Hence, SPP-TRC8 mediated abundance control of SQS acts as a metabolic switch within the mevalonate pathway.

\section{Introduction}

Cellular levels of key metabolites including lipids and sterols need to be tightly regulated in order to match the cellular demand. In particular, cholesterol, the most abundant individual lipid, has profound influence on membrane properties. Thus, cholesterol levels are modulated by uptake, endogenous synthesis, intracellular storage as cholesteryl ester, and efflux to apolipoproteins that altogether maintain membrane cholesterol homeostasis and prevent deleterious effects of hypercholesterolemia. Cholesterol is synthesized in the Endoplasmic Reticulum (ER) by the mevalonate pathway in a multistep process (Goldstein \& Brown, 1990). One of the intermediates of cholesterol biosynthesis, farnesyl pyrophosphate (FPP), serves also as the building block of several additional molecules including dolichol and ubiquinone and it is utilized in post-translational modifications such as glycosylation and prenylation (Edwards \& Ericsson, 1999). Since these isoprenoids affect important biological activities, commitment to the non-sterol branch of the mevalonate pathway needs to be tightly controlled. On one side, enzymes of the mevalonate pathway are transcriptionally upregulated by the sterol regulatory element binding protein (SREBP) in response to low cholesterol (Brown et al., 2018). On the other hand, rate-limiting mevalonate pathway enzymes, HMG-CoA reductase (HMGCR) and squalene epoxidase (SQLE), are under posttranslational feedback inhibition (Foresti et al., 2013, Gill et al., 2011, Schumacher \& DeBose-Boyd, 2021, Zelcer et al., 2014). When there is no demand for synthesis, the abundance of these key enzymes is downregulated by the ER-associated degradation (ERAD) pathway, a mechanism whereby proteins are targeted for turnover by the proteasome (Lemberg \& Strisovsky, 2021). While these homeostatic mechanisms determine 
the overall biosynthesis rate, SQLE has been suggested to influence the flux between the sterol- and non-sterol arm of the mevalonate pathway (Foresti et al., 2013, Gill et al., 2011, Zelcer et al., 2014) (Fig EV1A). However, also a truncated SQLE form exists (SQLE-S) that persist cholesterol-induced degradation (Coates et al., 2021), posing the question of how pathway selection is accommodated. Underlying most of these homeostatic responses to fluctuating amounts of cholesterol is the sensing of its level by a number of regulatory ER membrane proteins via conserved sterol-sensing domains (SSD) (Howe et al., 2016). At low cholesterol level, SSD-mediated protein-protein interactions are destabilized enabling trafficking of the SREBP from the ER to the Golgi, where its transcription factor domain is released and activated by regulated intramembrane proteolysis (Rawson et al., 1997). Conversely, under conditions of high cholesterol, HMGCR is targeted to a redundant set of ERAD E3 ubiquitin ligases, leading to its extraction from the membrane and proteasomal turnover (Lemberg \& Strisovsky, 2021). In addition to this ERAD dislocation mechanism that is exemplified by the E3 ubiquitin ligase Hrd1, cells have a non-canonical proteolytic arm of the ERAD pathway that is centered around signal peptide peptidase (SPP) (Avci \& Lemberg, 2015). SPP is an aspartic intramembrane protease related to Alzheimer's disease-associated $\gamma$-secretase (Weihofen et al., 2002). In addition to its role in processing signal peptides, SPP is also involved in protein degradation, functionally interacting with the ERAD E3 ubiquitin ligases TRC8 and MARCH6 (Boname et al., 2014, Chen et al., 2014, Stagg et al., 2009, Stefanovic-Barrett et al., 2018). However, the mechanism of regulation and the full impact of SPP-triggered degradation on cellular physiology have not been resolved yet. Here we show that SPP and TRC8 trigger cholesterol-dependent proteasomal degradation of squalene synthase (SQS), thereby serving as a metabolic switch at the branching point of the mevalonate pathway. 


\section{Results}

Substrate proteomics identifies SQS as a physiological SPP substrate

To identify endogenous substrates for SPP, we compared the proteome of ER-derived microsomes from Hek293T cells, with or without treatment with the SPP inhibitor (Z-LL) $2^{-}$ ketone (Weihofen et al., 2002), using stable-isotope labeling by amino acids in cell culture (SILAC) and quantitative proteomics (Fig 1A). We identified 15 ER-resident proteins that showed an increased steady-state level of more than $20 \%$ in the inhibitor treated condition (see Table EV1). In addition to the previously identified SPP substrates heme oxygenase 1 (HO1) and syntaxin-18 (Avci et al., 2019, Boname et al., 2014, Hsu et al., 2015), inhibition of SPP led to an increased level of SQS (Fig 1B), an ER-resident tail-anchored protein that defines the critical branching point of the sterol arm of the mevalonate pathway, by converting FPP into squalene (Fig EV1A) (Goldstein \& Brown, 1990). In contrast, 69 additional tail-anchored proteins identified were not significantly changed in their abundance (Table EV1), indicating that SPP is highly specific. Consistent with an SPP-mediated degradation of SQS, western blot analysis of $(Z-L L)_{2}$-ketone treated cells showed a significant increase of its steady-state level. In contrast, HMGCR level remained unchanged (Fig 1C). Furthermore, inhibition of SPP delayed SQS turnover in a cycloheximide chase assay (Fig 1D), confirming that SPP affects SQS stability. We tested release of the SPPinduced cleavage fragment into the cytoplasm by cellular fractionation, revealing the generation of a (Z-LL) $)_{2}$-ketone-sensitive SQS cleavage fragment migrating just below the fulllength form (Fig EV1B). In agreement with this, co-expression of a FLAG-tagged SQS construct with SPP destabilized the full-length form and a faster migrating band was observed. In contrast, ectopic expression of the catalytically inactive mutant SPP ${ }^{\mathrm{D} 265 \mathrm{~A}}$ had no effect (Fig 1E). The tail-anchored protein Ubc6 was completely resistant to SPP-catalyzed cleavage (Fig EV1C), supporting the notion that SPP is highly specific. A key determinant of SPP substrates are helix-destabilizing residues in the N-terminal portion of the transmembrane (TM) domain, as well as specific amino acid residues surrounding the cleavage site (Yucel et al., 2019). Mutation of either of two conserved TM serine residues 
(Fig EV1D) matching this substrate consensus completely stabilized SQS in the ER (Fig EV1E, F), indicating that a membrane-integral degradation signal (degron) determines its recognition by SPP. In order to confirm such a direct substrate-enzyme interaction, we performed co-immunoprecipitation experiments with the dominant-negative SPP ${ }^{\mathrm{D} 265 \mathrm{~A}}$ mutant (Avci et al., 2019, Chen et al., 2014), revealing efficient trapping of endogenous SQS, whereas the ER protein CLIMP63 did not interact (Fig EV1G). Taken together, these result show that SQS is a substrate for the SPP-dependent non-canonical ERAD pathway. Consistent with a physiological function of this abundance control mechanism, tamoxifeninduced SPP knockout in mouse bone marrow-derived dendritic cells led to a significant increase in steady state levels of SQS (Fig 1F). In contrast, SQLE, the enzyme downstream of SQS (Fig EV1A), was not affected by SPP ablation. Taken together with our proteomic analysis in Hek293T cells, this result indicates that the SPP-mediated abundance control mechanism specifically affects SQS, which is C-terminally anchored in the ER membrane, and no other mevalonate pathway enzymes that show other membrane topologies.

SQS is targeted for degradation by Hrd1 as well the SPP-TRC8-dependent ERAD pathway In order to investigate how SQS turnover relates to the SPP-specific ERAD machinery, we generated SPP knockout Hek293T cells. Although non-conditional SPP knockout mice die after embryonic day 13.5 (Aizawa et al., 2016), SPP-deficient tissue culture cells grow normal, while accumulating the unprocessed substrate $\mathrm{HO} 1$ and show a defect in processing of the hepatitis C virus core protein (Fig EV2A, B) (Aizawa et al., 2016, Boname et al., 2014). As observed in SPP-deficient primary murine dendritic cells (Fig 1F), we observed significant steady-state increase of endogenous SQS, but not HMGCR in Hek293T SPP knockout cells (Fig EV2C), whereas SQS mRNA level was not affected (Fig EV2D). Although there was still substantial turnover of SQS in SPP knockout cells, cycloheximide chase assay showed a significant increase of the half-life when compared to the wild type cells (Fig 2A). This result indicates that, SQS is subject to more than one ERAD pathway as had been observed for HMGCR (Fig EV1A). We next generated knockout cells of both E3 ubiquitin ligases that had 
been linked to SPP, namely TRC8 and MARCH6 (Chen et al., 2014, Stagg et al., 2009, Stefanovic-Barrett et al., 2018), and analyzed SQS protein stability and mRNA expression levels (Fig 2B and Fig EV2E). Similar to SPP knockout, we observed a partial stabilization of SQS in $\triangle T R C 8$ cells, whereas MARCH6 knockout did not show any significant effect (Fig 2B and Fig EV2F). Combined knockout of TRC8 and MARCH6 did not further stabilize SQS (Fig 2B). On the other hand, knockdown of the Hrd1 E3 ligase by siRNA transfection also interfered with SQS degradation (Fig EV2G). Strikingly, Hrd1 knockdown in the TRC8 and MARCH6 double-deficient cells almost completely stabilized SQS (Fig 2C). Consistent with SPP and TRC8 acting in one linear ERAD pathway, addition of the SPP inhibitor (Z-LL) $2^{-}$ ketone did not show any effect in $\triangle$ SPP or $\triangle T R C 8$ cells but stabilized SQS in $\triangle M A R C H 6$ cells (Fig EV2H-J). Taken together, these results show that SQS is subject to at least two independent branches of the ERAD pathway, the Hrd1-dependent canonical dislocation route (Lemberg \& Strisovsky, 2021) and the SPP-TRC8 pathway (Boname et al., 2014, Chen et al., 2014).

\section{Cholesterol activates SPP-TRC8 activity}

To assess whether SPP-TRC8-mediated SQS turnover is regulated, we studied its degradation rate in response to cholesterol levels. For this, we used lipoprotein-free medium and conditions blocking endogenous cholesterol synthesis, while synthesis of essential nonsterol isoprenoids is permitted and no toxicity was observed (Fig 3A and Fig EV3A) (Brown \& Goldstein, 1980, Nakanishi et al., 1988). SQS was significantly more stable in Hek293T cells grown in cholesterol-depletion medium when compared to cholesterol replete conditions (Fig 3B). The effect observed was comparable to cholesterol-induced ERAD of full-length SQLE (Fig 3B) (Zelcer et al., 2014), whereas the truncated SQLE-S form was stable, as observed before (Coates et al., 2021). In contrast, in SPP- or TRC8-deficient cells, SQS turnover was delayed and completely uncoupled from cholesterol levels, whereas cholesterol-induced ERAD of SQLE was unaffected by both knockouts (Fig 3C and D). This effect was specific to cholesterol, since 25-hydroxycholesterol, which serves as a potent trigger of HMGCR 
turnover (Sever et al., 2003), did not increase SPP-TRC8-dependent SQS degradation (Fig EV3B), as previously had been observed for SQLE (Gill et al., 2011). Taken together, our results show that for both enzymes a basal turnover exists. At high cholesterol conditions, SQLE is degraded by a MARCH6- and p97-dependent dislocation route (Zelcer et al., 2014), and SQS becomes subject to SPP-TRC8-triggered turnover. Consistent with a cholesterolinduced ubiquitination of SQS, we observed increased levels of several higher molecular weight SQS species in a ubiquitin-specific pulldown (Fig 3E). This difference was diminished in $\triangle T R C 8$ cells but still observed in the absence of SPP (Fig 3E). These results suggest that, TRC8-catalyzed ubiquitination serves as the sterol-sensitive step that is succeeded by SPPcatalyzed release from the ER membrane. We next asked whether cholesterol addition leads to an SQS-specific effect, or whether increased degradation rate is a consequence of general rise in SPP-TRC8 activity. Therefore, we studied degradation of the SPP substrate HO1 (Boname et al., 2014, Hsu et al., 2015) in the absence and presence of exogenously added cholesterol. Interestingly, HO1 turnover was also significantly accelerated in highcholesterol medium, compared to cholesterol-free conditions (Fig EV3C), whereas it was completely stabilized in SPP-deficient cells (Fig EV3D). This result indicates that the cholesterol-induced activation of SPP is likely a more general phenomenon.

\section{TRC8 triggers SPP-catalyzed SQS cleavage}

Since the TM domain of SQS and HO1 do not share any obvious sequence motif that might directly sense cholesterol levels, we asked whether the SPP-TRC8 complex acts as a sensor. Expression of TRC8 harboring a tyrosine-32-glutamate (Y32E) mutation, which previously has been suggested to compromise its SSD (Lee et al., 2010), showed significantly reduced activity in triggering SQS degradation in $\triangle T R C 8$ cells compared to the wild-type construct (Fig 4A and B). Interestingly, deletion of TRC8's catalytic RING domain (RING ${ }^{\mathrm{mt}}$ ) only partially abrogated rescue of the knockout phenotype (Fig 4A and C), indicating that TRC8 has also a non-catalytic function in SQS turnover by sensing the cholesterol level. Consistent with this hypothesis the non-catalytic function of TRC8-RING ${ }^{\mathrm{mt}}$ 
was entirely cholesterol dependent (Fig 4D) and the RING ${ }^{\text {mt }}$ Y $32 E$ double mutant of TRC8 did not rescue SQS degradation in $\triangle T R C 8$ cells (Fig 4A and E). In addition, SPP inhibition under high cholesterol conditions in $\triangle T R C 8$ cells expressing TRC8-RING ${ }^{\text {mt }}$ led to a significant stabilization of SQS level (Fig 4F), suggesting an allosteric activation of SPP proteolytic activity mediated through TRC8. Consistent with this hypothesis, we observed a subtle but significant increase of labeling with the SPP-specific activity-based probe HB-169 in microsomes isolated from cells grown in cholesterol rich conditions, compared to cholesterol depletion (Fig 4G and EV4A-B). Altogether, these results indicate that high cholesterol conditions enhance the proteolytic activity of SPP. Moreover, for complete SQS turnover, both the E3 ubiquitin ligase as well as the sterol sensing-activity of TRC8 are required.

SPP-mediated SQS degradation acts as a metabolic switch of the mevalonate pathway Next, we tested the influence of SPP on cellular cholesterol levels by analyzing the lipidome of wild type and $\triangle$ SPP Hek293T cells (Fig EV5A-E). In order to uncouple cellular cholesterol level from receptor-mediated uptake (Goldstein \& Brown, 1990), cells were grown in lipiddepleted medium. Total cholesterol levels were significantly increased in all three $\triangle$ SPP clones (Fig EV5F). The difference was even more pronounced in the lipidomic analysis upon subcellular fractionation, showing a striking increase of cholesterol in both endomembrane and plasma membrane fractions (Fig 5A). The levels of cholesterol in the cell needs to be tightly controlled and even small changes can have profound effects. As a way to test how ablation of SPP affects plasma membrane properties, we measured uptake of fluorescently labelled dextran from the medium. Consistent with cholesterol-dependent endocytosis (Sandvig et al., 2008), in Hek293T cells grown in rich medium 70-kDa dextran was visible in various vesicles, whereas no internalization was observed in cholesterol-depleted medium (Fig 5B). Strikingly, significantly more $70-k D a$ dextran was internalized in $\triangle$ SPP cells in the cholesterol depletion conditions (Fig 5B), indicating that increased plasma membrane cholesterol level, due to uncoupled cholesterol biosynthesis in $\triangle$ SPP cells, facilitates 
endocytosis under these conditions. This increased level of cholesterol-dependent endocytosis in $\triangle$ SPP cells was even more pronounced for the uptake of 10-kDa dextran (Fig 5B), which, in addition to macropinocytosis, also becomes subject to a less-well defined pinocytosis route (Sandvig et al., 2008). This shows that, SPP-mediated SQS abundance control is important to fine-tune cellular cholesterol levels with a broad impact on membrane properties. Total lipidome analysis of Hek293T wt and $\triangle$ SPP cells did not show any major differences in glycerophospholipid and storage lipid content. However, we observed an overall reduction in sphingolipid levels in $\triangle$ SPP cells (Fig EV5G), as well as an increase in long chain phosphatidylserine (36:1, see Fig EV5C) that has been linked to positioning of cholesterol in the plasma membrane (Skotland \& Sandvig, 2019). The mechanism of how SPP influences sphingolipid homeostasis and coupling of cholesterol to membrane composition remain important questions.

Turning back to the mevalonate pathway, our data suggest that SPP does not affect HMGCR (Fig 1C and Fig 2A) or SQLE (Fig 3C), but shows a specific effect on SQS. Since the SQScatalyzed conversion of two molecules of FPP to squalene determines to which extent the mevalonate pathway generates sterol or other biologically important non-sterol molecules including dolichol and ubiquinone, we suggest that it serves as a major metabolic switch at the branching point of the mevalonate pathway (Fig 5C). Consistent with this, we observed a significant reduction of dolichol phosphate levels in one $\triangle$ SPP cell clone (Fig 5D), which serves as the lipid intermediate for $\mathrm{N}$-linked glycosylation (Fig EV1A). Although the extent of the reduction is variable in different $\triangle \mathrm{SPP}$ clones, there may be compensating mechanisms in place, as small changes are predicted to have profound effects on several key cellular functions. Over all, we revealed a cholesterol regulated molecular switch at the branching point of the mevalonate pathway that can act as a fast-reacting fine-tuning process to control the flux of the FPP intermediate into two arms of the mevalonate pathway according to the cellular needs. How the complex cellular metabolism of non-sterol mevalonate products influences this switch and whether there are compensating mechanisms that suppress an 
SPP knockout phenotype, as well as how an SPP upregulation observed in cancer cells (Hsu et al., 2018, Wei et al., 2017) impacts this mechanism, remain to be important questions.

\section{Discussion}

Regulation of metabolite levels according to cellular needs is crucial to maintain homeostasis. Here we show that, the regulatory arm of the ERAD pathway (ERAD-R) centered around SPP (Avci \& Lemberg, 2015) acts as a so far unrecognized metabolic switch in the mevalonate pathway (Fig 6). While HMGCR, as the first rate-limiting enzyme of the multistep pathway, determines the overall flux of acetyl-CoA into isoprenoid biosynthesis, regulated turnover of SQS defines a metabolic switch that shunts FPP between the sterol and non-sterol branches. Cholesterol levels are increased in SPP knockout cells, due to increased SQS abundance, whereas a product of the non-sterol branch, dolichol phosphate, appears to be inversely regulated. This shows that, in addition to the control of the ratelimiting enzyme HMGCR by multiple ERAD E3 ubiquitin ligases (Gardner et al., 2001, Jiang et al., 2018, Jo et al., 2011, Zelcer et al., 2014), cholesterol also acts as a negative feedback regulator on the branching-point enzyme SQS. Consistent with this, we observed that the ERAD E3 ubiquitin ligases TRC8 and Hrd1 show an overlapping substrate spectrum, potentially allowing integration of different signals to control the abundance of SQS and ensuring plasticity of the response. The change in cholesterol levels in SPP knockout cells is also reflected to an increased capacity of cholesterol-dependent endocytosis compared to wild type cells, proving the physiological relevance of this metabolic switch.

We reveal that SPP is activated partially by a non-catalytic sterol-sensing function of TRC8 to cleave a specific set of tail-anchored proteins including SQS and HO1. This observation answers a long-standing question about the physiological relevance of the predicted SSD in TRC8 (Lee et al., 2010). Different to ERAD of HMGCR (Schumacher \& DeBose-Boyd, 2021) and activation of the transcription factor SREBP (Rawson et al., 1997), 25hydroxycholesterol does not show any effect on the SPP-TRC8-mediated protein abundance 
control mechanism. This difference indicates that, for this ERAD-R mechanism the end product of cholesterol biosynthesis is sensed and not the general flux through the mevalonate pathway is monitored. The molecular mechanism of how TRC8's SSD activates ERAD-R remains to be determined. However, we assume that recognition of single-pass TM substrates by the SPP-TRC8 complex is independent of SCAP/Insig adaptor proteins that are known to control HMGCR and SREPB (Hua et al., 1996). Since SPP specificity is determined by TM substrate features (Yucel et al., 2019), we suggest that the cholesterolinduced increase of the SQS processing rate is caused by allosteric activation of SPP by the membrane-integral SSD of TRC8. Presenilin, a mechanistically related aspartic intramembrane protease serving as the active subunit of the $\gamma$-secretase complex, is also modulated by cholesterol, which impacts generation of neurotoxic amyloid- $\beta$ peptides in Alzheimer's disease (Wahrle et al., 2002). A recent $\gamma$-secretase structure revealed three distinct cholesterol-binding sites in the non-catalytic subunit Aph-1 adjacent to presenilin (Yang et al., 2019), suggesting that allosteric regulation of aspartic intramembrane proteases by lipids is a more general principle. Overall, intramembrane proteolysis emerges as a fast and irreversible post-translational modification that controls the activity and abundance of various organellar membrane proteins (Avci \& Lemberg, 2015).

Human cancer association studies showed that SPP expression is increased in glioblastoma, lung, and breast cancer cells (Hsu et al., 2018, Wei et al., 2017). Based on our findings, we hypothesize that reduced SQS level upon increased SPP expression and concomitant increased flux of FPP into dolichol and ubiquinone synthesis may help to handle the increased metabolic load of rapidly growing cancer cells. Consistent with this idea, a recent study showed that synthesis through the mevalonate pathway is shifted towards ubiquinone to sustain viability of p53-deficient cancer cells exposed to metabolic stress (Kaymak et al., 2020). Likewise, heme metabolism may contribute to settle the microenvironment for cancer cell growth. These new insights in the role of SPP in cellular metabolism control offer the prospect of novel therapeutic intervention strategies. 


\section{Materials and Methods}

\section{Antibodies}

The following antibodies were used in this study: rabbit polyclonal anti-SPP (kind gift of C. Schaller), mouse monoclonal anti-FLAG (M2, Sigma-Aldrich, \#F1804), rat monoclonal antiHA (3F10, Sigma-Aldrich, \#11867423001), mouse monoclonal anti-CLIMP63 (G1/296, Enzo Life Sciences, \#ENZ-ABS669-0100), mouse monoclonal anti- $\beta$ actin (AC-15, Sigma-Aldrich, \#A1978), mouse monoclonal anti-ubiquitin (P4D1, Santa Cruz Biotechnology, \#sc-8017), rabbit polyclonal anti-HO1 (Enzo Life Sciences, \#ADI-SPA-896-F), rabbit polyclonal antiSQLE (Proteintech, \#12544-1-AP), rabbit monoclonal anti-SQS (Abcam, \#ab109723), rabbit monoclonal anti-SQS (Abcam, \#ab195046), mouse monoclonal anti-GFP (Sigma-Aldrich, \#11814460001), mouse monoclonal anti-HMGCR (Merck, \#MABS1233), rabbit polyclonal anti-Synoviolin (Hrd1) (Bethyl Laboratories, \#A302-946A), AlexaFluor488 goat anti-mouse $\lg G(H+L)$ (Invitrogen, \#A-11029), mouse monoclonal S-peptide epitope antibody (Thermo Fischer \# MA1-981). Custom-made polyclonal antisera were generated and affinity-purified against a synthetic peptide representing the C-terminus of murine SPP (Pineda AntikörperService, epitope: AETESKEESTEASASKRLEK).

\section{Plasmids}

Plasmids based on pcDNA3.1 (Invitrogen) encoding human SPP with a triple HA-tag inserted between residue 373 and the C-terminal KKEK ER-retention signal (SPP-HA) was described previously (Chen et al., 2014). The active-site mutant SPP D265A was introduced by sitedirected mutagenesis (Stratagene). Human SQS (FDFT1, Gene ID 2222, full-ORF Gateway cDNA clone 101218403) and Ubc6-FLAG (Gene ID 51465, IMAGE 8322482) with a Nterminal triple FLAG-tag were inserted as EcoRI/Xhol-fragment into pcDNA3.1. TM mutants of SQS were generated by site-directed mutagenesis. For the generation of doxycyclineinducible cell lines, we introduced constructs for human TRC8 (Gene ID 11236), TRC8RING ${ }^{\text {mt }}$ lacking residues 547 to 580 (gift from R. Gemill (Brauweiler et al., 2007)), and the 
Y32E SSD mutant generated by site-directed mutagenesis (this study), with a C-terminal single HA-tag respectively, into pcDNA5/FRT/TO (Invitrogen). Plasmids encoding an ERtargeting signal sequence fused to an RFP followed by a KDEL ER-retention signal (RFPKDEL) (Snapp et al., 2006) and the N-terminal 195 residues of the HCV polyprotein (Glasgow strain genotype 1a) corresponding to the core protein and 4 amino acids of the envelope protein E1 fused to an N-terminal triple FLAG-tag (Niemeyer et al., 2019) have been described before.

\section{Cell culture and transfection}

Hek293T and Hek293 Flp-In T-REx cells were maintained without antibiotics in Dulbecco's modified Eagle's medium (DMEM) (Invitrogen) supplemented with $10 \%$ fetal bovine serum (FBS) (Invitrogen) and grown as monolayers at $37^{\circ} \mathrm{C}$ and $5 \% \mathrm{CO}_{2}$. Hek293 Flp-In T-REx cells were additionally supplemented with $100 \mu \mathrm{g} / \mathrm{ml}$ hygromycin B (Invitrogen) and $10 \mu \mathrm{g} / \mathrm{ml}$ blasticidine (Gibco). Transient transfections were performed using 40-kDa linear polyethyleneimine (PEI MAX, Polysciences) (Durocher et al., 2002). Typically, $1 \mu \mathrm{g}$ encoding substrate and $500 \mathrm{ng}$ of plasmid encoding wt or mutant SPP were used per well of a 6-well plate. Total DNA ( $2 \mu \mathrm{g} /$ well) was held constant by adding empty plasmid. If not stated otherwise, cells were harvested $24 \mathrm{~h}$ after transfection. For inhibition of the proteasome and SPP, $2 \mu \mathrm{M}$ epoxomicin and $50 \mu \mathrm{M}(\mathrm{Z}-\mathrm{LL})_{2}$-ketone (Merck Millipore) were added from stock solutions in dimethyl sulfoxide (DMSO). As vehicle control, the same amount of DMSO was used. For the induction of $\mathrm{HO} 1,20 \mu \mathrm{M}$ hemin (ferriprotoporphyrin IX chloride, Sigma-Aldrich) was added to the cells from a $20 \mathrm{mM}$ stock solution in $0.05 \mathrm{M} \mathrm{NaOH}$ and incubated overnight. For immunofluorescence staining, Hek293T cells were seeded onto $12 \mathrm{~mm}$ cover slips in 24-well plates and transfected with $500 \mathrm{ng}$ plasmid encoding substrate and $150 \mathrm{ng}$ RFP-KDEL. Total amount of DNA ( $1 \mu \mathrm{g} /$ well) was held constant with empty plasmid.

\section{CRISPR/Cas9-mediated gene editing}


CRISPR sgRNA oligonucleotide sequences targeting SPP (HM13 gene), TRC8, or MARCH6 were designed using the E-CRISP online tool (Heigwer et al., 2014). The sgRNAs were cloned into the Bbsl-digested px459 (pSpCas9(BB))-2A-Puro V2.0, (Addgene \#62988) vector, verified by sequencing and used for transient transfection of Hek293T cells and Hek293 Flp-In T-REx cells. sgRNA-targeted cell populations were selected with $3 \mu \mathrm{g} / \mathrm{ml}$ puromycin and clonal cell lines were generated by limited dilution. Knockout clones for SPP were identified by immunoblotting and validated by sequencing of genomic DNA. Validation of TRC8 and MARCH6 knockout cells was based on indel detection. Identification of indels was performed using the online tools TIDE (Brinkman et al., 2014), CRISP-ID (Dehairs et al., 2016), and ICE v2 (Hsiau et al., bioRxiv, https://doi.org/10.1101/251082). The following sgRNAs were used: SPP-1 (clone \#1 and \#3) GCCCTCAGCGATCCGCATAA, SPP-2 (clone \#2) GCCTGAAACAATCACCAGCC, TRC8 CCAGACATACTACGAGTCTT, MARCH6 TATCATCCTTGTGTATGTAC. Genomic C-terminal tagging of SPP with S-tag is performed as described in Fueller et.al.2020. Genomic integration of the tagging cassette was tested by PCR and confirmed by sequencing and western blot analysis.

\section{Generation of stable Hek293 Flp-In T-REX cell lines}

For the generation of doxycycline-inducible stable cell lines, Flp-In T-REx Hek293ATRC8 cells were co-transfected with pOG44 (Invitrogen) and either pcDNA5/FRT/TO/TRC8, pcDNA5/FRT/TO/TRC8-RING ${ }^{\mathrm{mt}}$, or pcDNA5/FRT/TO/TRC8-Y32E and cells were selected with blasticidin (10 $\mu \mathrm{g} / \mathrm{ml}$, Gibco) and hygromycin B (100 $\mu \mathrm{g} / \mathrm{ml}$, Invitrogen).

\section{Mouse lines}

Mice with an inducible knockout of SPP were generated by crossing HM13 ${ }^{\mathrm{fl} / \mathrm{fl}}$ mice (EMMA, EM:08694) on a C57BL/6N background with R26-Cre-ERT2 mice

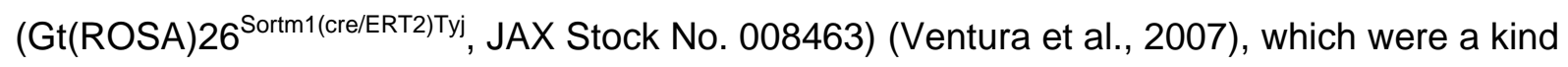
gift of Dr. Anthony Gavalas, TU Dresden. The following primers were employed for genotyping: HM13-5arm-WT-F: TGTAACAGCTCGCTTCGGAG, HM13-Crit-WT-R: 
TGAAATCAAGCCCAGGACCA; R26R.univF: AAAGTCGCTCTGAGTTGTTAT; CreER-R1: CCTGATCCTGGCAATTTCG. Breeding and handling of mice was approved by Landesdirektion Sachsen (DD24.1-5131/450/12, DD24.1-5131/449/55). All experiments were conducted using mice which were matched for age and sex.

\section{Generation of Bone marrow-derived dendritic cells (BMDC)}

Bone marrow was flushed from femur and tibia using BMDC medium (RPMI (Gibco) $+10 \%$ FCS $+100 \mathrm{U} / \mathrm{ml}$ penicillin (Sigma) $+100 \mu \mathrm{g} / \mathrm{ml}$ streptomycin (Sigma) $+50 \mu \mathrm{M} \beta-$ mercaptoethanol (Gibco)). Afterwards, cells were resuspended using a 23G cannula and passed through a $100 \mu \mathrm{m}$ cell strainer (Gibco). $5 \times 10^{6}$ cells were seeded in $10 \mathrm{ml}$ of BMDC medium supplemented with $20 \mathrm{ng} / \mathrm{ml}$ GM-CSF (Immunotools) and $1 \mu \mathrm{M} 4 \mathrm{OH}$-Tamoxifen (Sigma) in order to induce knockout of SPP. After 3 days, $10 \mathrm{ml}$ of BMDC medium supplemented with GM-CSF were added. On day 6 after seeding, $10 \mathrm{ml}$ of culture medium were replaced with $10 \mathrm{ml}$ of fresh BMDC medium containing $10 \mathrm{ng} / \mathrm{ml} \mathrm{GM-CSF}$. On day 8 , cells were harvested and processed for Western blot analysis.

\section{siRNA knockdown of Hrd1}

For the knockdown of Hrd1 (SYVN1 gene), cells were transfected with an ON-TRAGETplus siRNA smart pool (L-007090-00-0005) obtained from Dharmacon. As control, non-targeting pool siRNA (D-001810-01) was used. Cells were seeded onto poly-L-lysine (PLL)-coated 12well plates in DMEM supplemented with 10\% FBS and transfected using the RNAiMAX reagent (Life Technologies) according to manufacturer's instructions. After $24 \mathrm{~h}$, medium was exchanged to fresh medium containing FBS. Cells were harvested $48 \mathrm{~h}$ after transfection. The final molarity of siRNA per well was 20 picomol.

\section{Mass spectrometry-based substrate screen}

For the identification of SPP substrates, we used stable isotope labelling by amino acids in cell culture (SILAC)-based quantitative organelle proteomics. For this, Hek293T cells were 
grown for five doublings in medium supplemented with either heavy amino acids $\left({ }^{13} \mathrm{C}_{6}{ }^{15} \mathrm{~N}_{4}\right.$ - LArg and ${ }^{13} \mathrm{C}_{6}{ }^{15} \mathrm{~N}_{2}$-L-Lys; Silantes) or unlabelled amino acids before applying (Z-LL) $)_{2}$-ketone $(50 \mu \mathrm{M})$ or DMSO as vehicle control. For each condition ((Z-LL) $)_{2}$-ketone or DMSO), $3 \times 150$ $\mathrm{mm}$ plates were used.

If not stated otherwise, all steps were performed at $4^{\circ} \mathrm{C}$. At day of harvest, cells were washed with ice-cold 1x PBS (10 mM NaH${ }_{2} \mathrm{PO}_{4}, 1.4 \mathrm{mM} \mathrm{KH}_{2} \mathrm{PO}_{4}, 2.7 \mathrm{mM} \mathrm{KCl}, 140 \mathrm{mM} \mathrm{NaCl}, \mathrm{pH}$ 7.4), harvested in PBS-EDTA (1x PBS, 1 mM EDTA, pH 8.0, $0.2 \mathrm{~g} / \mathrm{l}$ glucose) and an equal number of cells were resuspended in ice-cold hypotonic buffer (10 mM HEPES-KOH, pH 7.4, $1.5 \mathrm{mM} \mathrm{MgCl}$, $10 \mathrm{mM} \mathrm{KOAc}, 0.5 \mathrm{mM}$ dithiothreitol (DTT)) containing $10 \mu \mathrm{g} / \mathrm{ml}$ phenylmethylsulfonyl fluoride (PMSF) and Complete EDTA-free protease cocktail (Roche). Cells were incubated on ice for $10 \mathrm{~min}$ and lysed by passing five times through a 27-Gauge needle, followed by centrifugation for $15 \mathrm{~min}$ at $400 \times \mathrm{g}$ to remove cell debris and nuclei. The supernatant was laid on top of a low salt sucrose cushion (500 mM sucrose, 50 mM HEPES$\left.\mathrm{KOH}, \mathrm{pH} 7.4,50 \mathrm{mM} \mathrm{KOAc}, 5 \mathrm{mM} \mathrm{Mg}(\mathrm{OAc})_{2}\right)$ and centrifuged for $20 \mathrm{~min}$ at 100,000 $\mathrm{g}$ in a 70Ti rotor (Beckman Coulter). The resulting membrane pellet was resuspended in $1 \mathrm{ml}$ rough microsome (RM) buffer (250 mM sucrose, 50 mM HEPES-KOH, pH 7.4, 50 mM KOAc, 2 mM $\mathrm{Mg}(\mathrm{OAc})_{2}, 1 \mathrm{mM}$ DTT $)$ and extracted by $500 \mathrm{mM} \mathrm{KOAc}$ and $50 \mathrm{mM}$ EDTA on ice for $15 \mathrm{~min}$, followed by centrifugation through a high salt sucrose cushion $(500 \mathrm{mM}$ sucrose, $50 \mathrm{mM}$ HEPES-KOH, pH 7.4, $\left.500 \mathrm{mM} \mathrm{KOAc,} 5 \mathrm{mM} \mathrm{Mg}(\mathrm{OAc})_{2}\right)$ for $20 \mathrm{~min}$ at 100,000 x g. The pellet thereof was resuspended in $1 \mathrm{ml}$ freshly prepared, ice-cold sodium carbonate (100 mM) buffer and transferred onto an alkaline sucrose cushion (125 mM sucrose, $100 \mathrm{mM} \mathrm{Na}_{2} \mathrm{CO}_{3}$ ) followed by centrifugation for 20 min at $100,000 \times g$. The pellet was resuspended in RM buffer and snap frozen in liquid nitrogen.

For proteomics, pellets in RM buffer were diluted in 2x SDS-sample buffer (see below), separated by sodium dodecyl sulfate (SDS) polyacrylamide gel electrophoresis SDS-PAGE for $1 \mathrm{~cm}$ and samples were excised from the gel before reducing with DTT and alkylation with 
iodoacetamide. Digestion with trypsin was done overnight at $37^{\circ} \mathrm{C}$, followed by quenching with $0.1 \%$ trifluoracetic acid (TFA; Biosolve, Valkenswward), and the supernatant was dried in a vacuum concentrator before LC-MS analysis. Nanoflow LC-MS ${ }^{2}$ analysis was performed using an Ultimate 3000 LC system coupled to an QExactive HF mass spectrometer (Thermo Scientific). Samples were dissolved in 0.1\% TFA and loaded onto a C18 Acclaim PepMap100 trap-column (Thermo Fisher Scientific) with a flow rate of $30 \mu \mathrm{l} / \mathrm{min} 0.1 \%$ TFA. Subsequently, peptides were eluted and separated on an C18 Acclaim PepMap RSLC analytical column $(75 \mu \mathrm{m} \times 250 \mathrm{~mm}$, Thermo Fisher Scientific) with a flow rate of $300 \mathrm{nl} / \mathrm{min}$ in a 120 min gradient of $3 \%$ buffer $A(0.1 \%$ formic acid $)$ to $40 \%$ buffer $B(0.1 \%$ formic acid/acetonitrile). The mass spectrometer was operated in data-dependent acquisition mode, automatically switching between $\mathrm{MS}$ and $\mathrm{MS}^{2}$. Collision induced dissociation $\mathrm{MS}^{2}$ spectra were generated for up to 20 precursors with normalized collision energy of $29 \%$. Processing of RAW files was performed using MaxQuant (v. 1.5.3.30). $\mathrm{MS}^{2}$ spectra were searched against the Uniprot human proteome database and the contaminants database by Andromeda search engine with the following parameters: Carbamidomethylation of cysteine residues, acetylation of protein $\mathrm{N}$ termini, and oxidation of Met were considered as variable modifications. Trypsin as the proteolytic enzyme with up to 2 missed cleavages was allowed. The maximum false discovery rate was 0.01 and a minimum peptide length of 7 amino acids was required. All other parameters were default parameters of MaxQuant. Quantitative normalized ratios were calculated by MaxQuant and used for further data analysis.

\section{Lipidomics}

In order to determine the change in lipid composition, Hek293T wt cells together with three different Hek293T $\Delta$ SPP clones were analyzed by lipidomics. At day 0, cells were seeded onto PLL-coated 100 mm dishes in DMEM supplemented with $10 \%$ FBS. At day 1 , cells were first washed two times with 1x PBS followed by sterol-depletion in medium containing DMEM supplemented with 5\% lipoprotein deficient serum (LPDS) (see below). At day of harvest (day 2 after seeding), membrane fractions were prepared by subcellular fractionation 
(see below). The last centrifugation step was performed for 60 min at $21,000 \times g$ at $4^{\circ} \mathrm{C}$ in a table-top centrifuge. Membranes were resuspended in methanol and subjected to lipid extractions using an acidic Bligh \& Dyer, except from plasmalogens, which were extracted under neutral conditions as described in (Ozbalci et al., 2013). Lipid standards were added prior to extractions, using a master mix containing phosphatidylcholine (13:0/13:0, 14:0/14:0, 20:0/20:0; 21:0/21:0; Avanti Polar Lipids, Alabaster, AL, USA), sphingomyelin (SM, d18:1 with $\mathrm{N}$-acylated 13:0, 17:0, 25:0, semi-synthesized (Ozbalci et al., 2013)), $\mathrm{D}_{6}$-cholesterol (Cambridge Isotope Laboratory), phosphatidylinositol (PI, 16:0/16:0 and 17:0/20:4; Avanti Polar Lipids, Alabaster, AL, USA), phosphatidylethanolamine (PE), phosphatidylserine (PS) and phosphatidylglycerol (PG) (14:1/14:1, 20:1/20:1, 22:1/22:1, semi-synthesized (Ozbalci et al., 2013), diacylglycerol (DAG, 17:0/17:0, Larodan), cholesterol ester (CE, 9:0, 19:0, 24:1, Sigma-Aldrich, St. Louis, MO, USA), triacylglycerol (TAG, $D_{5}-\mathrm{mix}, \mathrm{LM}-6000 / \mathrm{D}_{5^{-}}$ 17:0,17:1,17:1; Avanti Polar Lipids, Alabaster, AL, USA), ceramide (Cer) and glucosylceramide (HexCer) (both d18:1 with N-acylated 15:0, 17:0, 25:0, semisynthesized(Ozbalci et al., 2013), lactosylceramide (LacCer, d18:1 with N-acylated C12 fatty acid; Avanti Polar Lipids, Alabaster, AL, USA), phosphatidic acid (PA, 17:0/20:4; Avanti Polar Lipids, Alabaster, AL, USA), and lyso-phosphatidylcholine (LPC, 17:1; Avanti Polar Lipids, Alabaster, AL, USA). PE plasmalogen (PE P-)-containing standard mix was supplemented with PE P-mix 1 (16:0p/15:0, 16:0p/19:0, 16:0p/ 25:0), PE P-mix 2 (18:0p/15:0, 18:0p/19:0, 18:0p/25:0), and PE P-Mix 3 (18:1p/15:0, 18:1p/19:0, 18:1p/25:0). Semi-synthesis of PE Pwas performed as described in (Paltauf \& Hermetter, 1994). Lipid extracts were resuspended in $60 \mu \mathrm{l}$ methanol and samples were analyzed on an AB SCIEX QTRAP 6500+ mass spectrometer (Sciex, Framingham, MA, USA) with chip-based (HD-D ESI Chip; Advion Biosciences, Ithaca, NY, USA) nano-electrospray infusion and ionization via a Triversa Nanomate (Advion Biosciences, Ithaca, NY, USA) as previously described (Paltauf \& Hermetter, 1994). Resuspended lipid extracts were diluted 1:10 in 96-well plates (Eppendorf twin tec 96, colorless, Z651400-25A; Sigma-Aldrich, St. Louis, MO, USA) prior to measurement. Lipid classes were analyzed in positive ion mode applying either specific 
precursor ion (PC, lyso-PC, SM, cholesterol, Cer, HexCer, Hex2Cer, and PE-P) or neutral loss (PE, PS, PI, PG, and PA) scanning as described in(Paltauf \& Hermetter, 1994). Data evaluation was performed using LipidView (RRID: SCR_017003; Sciex, Framingham, MA, USA) and an in-house-developed software (ShinyLipids).

For dolichol phosphate analysis, cells were resuspended in $1 \mathrm{ml}$ methanol, then $200 \mathrm{pmol}$ of C55-DolP (Larodan, Sweden) was added as internal standard, followed by $1 \mathrm{ml} \mathrm{H}_{2} \mathrm{O}$. Alkaline hydrolysis and subsequent partitioning were performed as described before (Haeuptle et al., 2010) with few modifications. Briefly, the cell suspension was hydrolyzed by addition of 0.5 $\mathrm{ml} 15 \mathrm{M} \mathrm{KOH}$ and heated at $85^{\circ} \mathrm{C}$ for $60 \mathrm{~min}$. Phase partitioning was induced by the addition of $1 \mathrm{ml}$ methanol and $4 \mathrm{ml}$ dichloromethane and residual dolichyl esters were further hydrolyzed at $40^{\circ} \mathrm{C}$ for $60 \mathrm{~min}$. The lower phase was washed four times with $2.9 \mathrm{ml}$ dichloromethane / methanol / $\mathrm{H}_{2} \mathrm{O}(3: 48: 47, \mathrm{v} / \mathrm{v} / \mathrm{v})$ and evaporated to dryness. For methylation, dried samples were dissolved in $200 \mu$ ldichloromethane:methanol $(6.5: 5.2, \mathrm{v} / \mathrm{v})$ and then methylated with $10 \mu \mathrm{l}$ trimethylsilyldiazomethane (Sigma-Aldrich). After $40 \mathrm{~min}, 1 \mu \mathrm{l}$ acetic acid was added to neutralize an excess of trimethylsilyldiazomethane. LC-MS/MS analysis was performed on an Ultimate 3000 (Dionex) UHPLC system coupled to a QExactive HRMS (Thermo Scientific, MA, USA) in ESI positive ionization mode. For LC separations isopropanol/acetonitrile $(90: 10, \mathrm{v} / \mathrm{v})$ with $1 \mathrm{mM}$ ammonium acetate and $0.1 \%$ formic acid (mobile phase A) and acetonitrile/ $\mathrm{H}_{2} \mathrm{O}(60: 40$, v/v) with $1 \mathrm{mM}$ ammonium acetate and $0.1 \%$ formic acid (mobile phase B) were used. Dried samples were reconstituted in $40 \%$ mobile phase B. Samples were loaded on a CSH C18 column (150 x $1 \mathrm{~mm}$ inner diameter, particle size $1.7 \mu \mathrm{m}$; Waters) coupled to a QExactive mass spectrometer. The flow rate was $0.1 \mathrm{ml} / \mathrm{min}$. The following gradient profile was employed: 0-3 $\mathrm{min}, 40-50 \% \mathrm{~B}, 3-9 \mathrm{~min}, 50$ 54\% B, 9-9.1 min, 54-70\% B, 9-17 min, 70-90\% B, 9.1-22 min, 90\% B, 22-22.5 min, 9040\% B, 22.5-30 min, 40\% B. Samples were automatically injected via the Dionex Ultimate 3000 LC system. Spray voltage was set to $4000 \mathrm{~V}$ with a capillary temperature of $320^{\circ} \mathrm{C}$. Full MS scans (m/z 790-1580 Da) were obtained with automatic gain control target of $3 \times 10^{6}$ ions 
and maximal injection time of $200 \mathrm{~ms}$. Data evaluation was performed using XCalibur (Thermo Fisher Scientific).

\section{Quantitative real-time PCR}

Quantitative real-time PCR was used to assess the SQS mRNA level (transcribed from the FDFT1 gene) in Hek293T wt cells or cells deficient for SPP, TRC8, MARCH6, or TRC8/MARCH6-double deficient cells. Therefore, RNA was isolated from confluent cells from one well of a 6-well plate with the NucleoSpin RNA isolation kit (Macherey-Nagel) according to manufacturer's protocol. $2 \mu \mathrm{g}$ RNA were reverse transcribed using the RevertAid First Strand cDNA Synthesis Kit (Thermo Scientific). Quantitative PCR was performed using the SensiFAST SYBR No-ROX kit (Bioline) according to the manufacturer's protocol and the LightCycler480 Instrument II (Roche) with the following cycle settings: preincubation for $2 \mathrm{~min}$ at $95^{\circ} \mathrm{C}$, followed by 40 cycles amplification at $95^{\circ} \mathrm{C}$ for $5 \mathrm{sec}, 57^{\circ} \mathrm{C}$ for $10 \mathrm{sec}$, and $72^{\circ} \mathrm{C}$ for $25 \mathrm{sec}$. Each reaction was performed in technical triplicate. TATAA-box binding protein (TBP) and $\beta-2$ microglobulin $(\beta 2 \mathrm{M})$ were used as reference genes. Primers used for TBP: 5'-CCGGCTGTTTAACTTCGTT and 5'-ACGCCAAGAAACAGTGATGC; $\beta 2 M$ : 5'-CACGTCATCCAGCAGAGAAT and 5'-TGCTGCTTACATGTCTCGAT; SQS/FDFT1: 5'GAGGACTTCCCAACGATCTCC and 5'-AACTCTGCCATCCCAATGCC. Relative quantification of gene expression was calculated as described previously (Pfaffl, 2001).

\section{Preparation of LPDS, cholesterol inclusion complexes and cholesterol-depletion and}

\section{repletion assay}

For the manipulation of the cellular cholesterol content, Hek293T cells were pre-treated and treated with DMEM containing 5\% LPDS. LPDS was prepared from FBS by density gradient ultracentrifugation using solid potassium bromide according to (Goldstein et al., 1983) with minor modifications. The density adjusted FBS was subjected to ultracentrifugation for $25 \mathrm{~h}$ at $310,500 \times g$ at $10^{\circ} \mathrm{C}$ in a $70 \mathrm{Ti}$ rotor (Beckman Coulter) before dialysis. The dialyzed fraction was sterile filtered and frozen at $-20^{\circ} \mathrm{C}$. 
Cholesterol repletion was carried out by incubating cells in the presence of cholesterol/methyl- $\beta$-cyclodextrin inclusion complexes (Chol/CD) which were prepared with minor modifications according to (Klein et al., 1995). Cholesterol (Sigma-Aldrich) was dissolved in isopropanol:chloroform (2:1) and added in small aliquots to a stirring $5 \%(\mathrm{w} / \mathrm{v})$ methyl- $\beta$-cyclodextrin (Sigma-Aldrich) solution in a water bath heated to $80^{\circ} \mathrm{C}$. The solution was cooled down, sterile filtered and kept at $4^{\circ} \mathrm{C}$ protected from light until use. The cholesterol to methyl- $\beta$-cyclodextrin molar ratio was $10: 1$.

For experiments involving sterol depletion and repletion, cells were seeded onto PLL-coated plates. After 24 hours, cells were washed two times with 1x PBS before switching to steroldepletion medium containing 5\% LPDS, supplemented with $5 \mu \mathrm{M}$ mevastatin and $50 \mu \mathrm{M}$ mevalonate (Sigma-Aldrich). After overnight sterol depletion in this medium, cells were treated with fresh sterol-depletion medium either in absence or in presence of $30 \mu \mathrm{g} / \mathrm{ml}$ Chol/CD complexes or other test agents.

\section{Cristal violet cytotoxicity assay}

For crystal violet cell viability assay, cells were seeded onto PLL-coated 24-well plates, sterol-depleted overnight with medium containing LPDS, mevastatin and mevalonate and treated with or without $30 \mu \mathrm{g} / \mathrm{ml}$ Chol/CD. At three hours after treatment, cells were washed with $1 \times$ PBS and fixed for $10 \mathrm{~min}$ at $4^{\circ} \mathrm{C}$ with cold methanol/acetone $(1: 1 ; 1 \mathrm{ml} /$ well). Cells were stained with $0.1 \%$ crystal violet in $25 \%$ methanol for 15 min followed by washing four times with tap water. Afterwards, the stained cells were air dried overnight and representative images were acquired using a Zeiss Axio Observer Z1 widefield microscope equipped with a LD-Plan-NEOFLUAR 20x/0.4 Air Ph2 objective at 20x magnification. For quantitative measurement, adsorbed dye was solubilized with methanol containing $0.1 \%$ SDS by gentle agitation for two hours. Optical density at $570 \mathrm{~nm}\left(\mathrm{OD}_{570}\right)$ from three biological replicates and each replicate in technical duplicate was measured using the Infinite M1000 microplate 
reader (Tecan Trading AG, Switzerland) and accompanying software i-control (v.1.10.4.0). For quantification, the mean OD for each cell line and condition tested was corrected by subtracting the mean $\mathrm{OD}_{570}$ of blank wells. The $\mathrm{OD}_{570}$ of FBS-treated cells for each cell line was set to 1.0 and the respective fold-change under treatment conditions for each cell lines was calculated.

\section{Cycloheximide chase experiments}

Cycloheximide $(100 \mu \mathrm{g} / \mathrm{ml})$ chase was performed $24 \mathrm{~h}$ after transfection of Hek293T cells or after doxycycline induction of Hek293 Flp-In T-REx cells. For chase of endogenous proteins, cycloheximide was added $48 \mathrm{~h}$ after seeding of Hek293T cells. Following the addition of the compound in medium onto cells, lysates were prepared at indicated time points and protein abundance was analyzed by western blotting. Time point 0 was immediately harvested and did not receive cycloheximide.

\section{Subcellular fractionation}

In order to isolate cellular membranes, subcellular fractionation was performed. Therefore, cells from 6-well or $100 \mathrm{~mm}$ dishes were washed with 1x PBS followed by adding $1 \mathrm{ml}$ PBSEDTA to detach cells. Cells were centrifuged for $10 \mathrm{~min}$ at $400 \times g$ at $4^{\circ} \mathrm{C}$, the supernatant was removed, and the cell pellet was resuspended in $1 \mathrm{ml}$ hypotonic buffer supplemented with $10 \mu \mathrm{g} / \mathrm{ml}$ PMSF and protease inhibitor cocktail (Roche). Cells were incubated on ice for $10 \mathrm{~min}$, lysed by passing 5 times through a 27-Gauge needle, followed by centrifugation for $5 \mathrm{~min}$ at $1,000 \times g$ at $4^{\circ} \mathrm{C}$ to pellet cells and debris. To pellet membranes, the post-nuclear supernatant was centrifuged for $30 \mathrm{~min}$ at $100,000 \times \mathrm{g}$ at $4^{\circ} \mathrm{C}$ using a S120AT2 rotor (Beckman Coulter). The resulting pellet containing the membranes was resuspended in SDS sample buffer (see below). 
Fractionation into plasma membrane and the ER-containing endomembrane fraction for the lipidome analysis was performed using the Minute ${ }^{\mathrm{TM}}$ Plasma Membrane Protein Isolation and Cell Fractionation Kit (Invent Biotechnologies, Inc.) according to manufacturer's instructions.

\section{Synthesis of SPP-specific probe HB-169}

N-Fluorenyl-9-methoxycarbonyl (Fmoc)-protected Sieber amide resin (100 mg, $80 \mu \mathrm{mol})$ was treated with $20 \%$ piperidine in N,N-dimethylformamide (DMF) for 5 min to swell the resin and deprotect the Fmoc group. Next, the resin was washed three times with DMF. All following Fmoc deprotections were performed according to this procedure. Fmoc-N'-methyltrityl-Llysine, two Fmoc-6-aminohexanoic acid, Fmoc-Benzoyl-L-Phenylalanine and Fmoc-LLeucine were subsequently coupled after Fmoc deprotection by adding amino acid (2 eq), Nhydroxybenzotriazole (HOBt) (2 eq) and N,N'-diisopropylcarbodiimide(DIC) (2 eq) to give intermediate 2 (see Fig EV4A). After the next Fmoc deprotection, the free amino group was coupled to building block 3, which was prepared according to literature procedures (Chun et al., 2004, Crump et al., 2012, Nadin et al., 2001). To this end, the carboxylic acid of 3 (2 eq) was preactivated for $30 \mathrm{~min}$ with 1 -[Bis(dimethylamino)methylene]-1H-1,2,3-triazolo[4,5b]pyridinium 3-oxide hexafluorophosphate (HATU) (1.95 eq) and N,N-diisopropylethylamine (DIPEA) (3 eq) and added to the resin. The mixture was shaken overnight at $30^{\circ} \mathrm{C}$. Next, the resin was treated with a $0.1 \mathrm{M}$ solution of tetrabutylammonium fluoride (TBAF) in tetrahydrofuran (THF) for 1 hour to remove the silyl group. Upon treatment with $1 \%$ trifluoroacetic acid (TFA) in dichloromethane (DCM) and solvent removal, intermediate 4 was purified by HPLC on a Prominence Ultra-fast Liquid Chromatography system (Shimadzu) using a Waters X-bridge $150 \mathrm{~mm} \mathrm{C18}$ prep column with a gradient of acetonitrile in water (with $0.1 \%$ TFA) over 32 min. Intermediate 4 was obtained as a white powder in a $1 \%$ yield over all steps. ESI-MS calculated for $\mathrm{C}_{70} \mathrm{H}_{96} \mathrm{~N}_{8} \mathrm{O}_{11}\left[\mathrm{M}+\mathrm{H}^{+}\right] \mathrm{m} / \mathrm{z}$ : 1131.68, found 1131.50 . Product $4(0.6 \mu \mathrm{mol}, 1 \mathrm{eq})$ was then coupled to 5-hexynoic acid $(1.1 \mathrm{eq})$ by reaction with HOBt (1.1 eq) and 1-ethyl-3-(3-dimethylaminopropyl)carbodiimide (EDC) (1.1 eq) in DMF. The crude reaction was purified by HPLC to give the final product HB169 (5) as a white solid 
in $48 \%$ yield. ESI-MS calculated. for $\mathrm{C}_{64} \mathrm{H}_{90} \mathrm{~N}_{8} \mathrm{O}_{10}\left[\mathrm{M}+\mathrm{H}^{+}\right] \mathrm{m} / \mathrm{z}: 1225.72$, found 1225.35. LCMS spectra were recorded on a Prominence Ultra-fast Liquid Chromatography system (Shimadzu) using a Waters X-bridge $2.1 \mathrm{~mm} \mathrm{C18}$ column with a gradient of $5 \%-80 \%$ acetonitrile in water (with $0.1 \%$ formic acid) over $22 \mathrm{~min}$.

\section{Labeling SPP-S tag with HB169 probe in microsomes}

$0.5 \mu \mathrm{L}$ of a $10 \mu \mathrm{M}$ DMSO stock solution of probe HB-169 (100 nM final concentration) was added to $50 \mu \mathrm{L}$ membrane fraction in RM buffer and the solution was mixed by pipetting up and down. For competition with (Z-LL) $)_{2}$-ketone, $0.5 \mu \mathrm{L}$ of a $1 \mathrm{mM}$ DMSO stock solution of $(Z$ $\mathrm{LL})_{2}$-ketone was added (10 $\mu \mathrm{M}$ final concentration) prior to addition of the probe. Samples were irradiated on ice for 30 min using a UVPTM Blak-Ray B-100AP high intensity UV lamp (230 V, $100 \mathrm{~W})$. Samples were then diluted $10 \mathrm{X}$ in solubilisation buffer $(450 \mu \mathrm{L}, 50 \mathrm{mM}$ HEPES pH $7.4+1 \%$ CHAPS + PI cocktail tablet) and solubilized at $4{ }^{\circ} \mathrm{C}$ for $1 \mathrm{~h}$ on a rotating wheel. Insoluble material was pelleted by centrifugation at $15,000 \times \mathrm{g}$ for $10 \mathrm{~min}$, and the supernatant containing solubilized membrane proteins was transferred to a separate Eppendorf tube. Click reagents were added: $2.5 \mu \mathrm{L}$ of 5 mM TAMRA-N3 in DMSO (25 $\mu \mathrm{M}$ final concentration), $5 \mu \mathrm{L}$ of $5 \mathrm{mM}$ THPTA in DMSO (50 $\mu \mathrm{M}$ final concentration), $10 \mu \mathrm{L}$ of $\mathrm{CuSO}_{4}$ in water and $10 \mu \mathrm{L}$ of sodium ascorbate in water (both freshly prepared before use, $500 \mu \mathrm{M}$ final concentration). The mixture was incubated for $1 \mathrm{~h}$ at ambient temperature with rotation. A $50 \mu \mathrm{L}$ portion was removed and precipitated overnight at $-20^{\circ} \mathrm{C}$ by the addition of $200 \mu \mathrm{L}$ acetone. The precipitated protein was obtained by centrifugation at $5000 \times \mathrm{g}, 1 \mathrm{~min}$ then at $17,000 \times g$ for $5 \mathrm{~min}$. The pellet was washed once with acetone $(200 \mu \mathrm{L})$, then airdried for $5 \mathrm{~min}$ before addition of $2 \mathrm{X}$ sample buffer and heating at $65^{\circ} \mathrm{C}$ for $10 \mathrm{~min}$. Samples were run on a 12\% SDS-PAGE gel and fluorescence was imaged on a Typhoon FLA 9500 (590 nm, TAMRA channel). S-tagged SPP was detected by Western blotting using an anti speptide primary antibody (MA1-981, Invitrogen) and anti-mouse-HRP (HAF007, R\&D systems) secondary antibody. 


\section{Fluorescence microscopy and fluid phase uptake assay}

For immunofluorescence staining, cells were first carefully washed with 1x PBS followed by fixation with $0.5 \mathrm{ml} /$ well $4 \%$ formaldehyde in PBS for 20 min with gentle agitation. Afterwards, cells were washed three times with $1 \times$ PBS and then permeabilized with $0.2 \mathrm{ml} / \mathrm{well} 0.5 \%$ Triton X-100 in PBS for 10 min at $4^{\circ} \mathrm{C}$. Following washing with $1 \times$ PBS (three times), unspecific binding sites were blocked by incubating the cells with blocking buffer (20\% FBS in 1 X PBS) for 30 min. Primary antibody in blocking buffer supplemented with $0.01 \%$ Triton X100 was applied for $1 \mathrm{~h}$ with gentle agitation followed by washing three times with $1 \mathrm{x}$ PBS, 5 min each. After this, the secondary antibody at respective dilution in blocking buffer was applied onto the cells and incubated for $1 \mathrm{~h}$ with agitation. From this step onwards, cells were protected from light to prevent antibody bleaching. Cells were washed three times with $1 \mathrm{x}$ PBS, 5 min each and mounted in Fluoromount-G (Southern Biotech). Confocal analysis was performed on a Zeiss LSM 780 inverted microscope. Lasers used were a $458 \mathrm{~nm}, 488 \mathrm{~nm}$, $514 \mathrm{~nm}$ Argon laser $(25 \mathrm{~mW})$ and a $561 \mathrm{~nm}$ DPSS laser $(20 \mathrm{~mW})$. Images were acquired using a Plan-APOCHROMAT 63x/1.4 oil objective at 1024 x 1024 pixels with line average 4 and a pinhole size of 1 Airy unit using the ZEN 2012 (v. 1.1.2.0) software. For image stacks, series of $8-12$ sections with $0.5 \mu \mathrm{m}$ along the Z-axis were taken. Images were processed and maximum intensity projections created using Fiji.

Fluid phase uptake was determined by incubating cells with $10 \mathrm{kDa}$ dextran-Alexa568 and $70 \mathrm{kDa}$ dextran-Oregon Green at $0.1 \mathrm{mg} / \mathrm{ml}$ each for $30 \mathrm{~min}$ in regular DMEM or medium supplemented with 5\% LPDS (see above). Subsequently, cells were washed two times with ice cold PBS and fixed with cold 4\% formaldehyde in PBS for 15 min. After fixation, cells were washed in PBS, stained with $5 \mu \mathrm{g} / \mathrm{ml}$ Hoechst 33342 stain in PBS for $5 \mathrm{~min}$, and finally rinsed with PBS prior to microscopy. Cellular uptake of fluorescently labelled dextran was analyzed by confocal microscopy using a Zeiss LSM 780 inverted microscope and quantified using Fiji/lmageJ (http://rsb.info.nih.gov/ij/) according to (Palm et al., 2017). 


\section{Immunoprecipitation and TUBE-ubiquitin pulldown}

For immunoprecipitation, cells were washed once with 1x PBS followed by treatment with the membrane-permeable crosslinker DSP $(2 \mathrm{mM})$ in PBS for 30 min on ice. Tris- $\mathrm{HCl}, \mathrm{pH} 7.4$ was added to a final concentration of $20 \mathrm{mM}$ for $15 \mathrm{~min}$ to quench the cross-linking reaction. Cells were washed with 1x PBS, harvested in PBS-EDTA followed by centrifugation for 3 min at $900 \times g$ at $4^{\circ} \mathrm{C}$. The supernatant was discarded, and the pellet was lysed in $1 \mathrm{ml}$ solubilization buffer (20 mM HEPES-KOH, pH 7.4, $150 \mathrm{mM} \mathrm{NaCl}, 1.5 \mathrm{mM} \mathrm{MgCl} 2,1 \mathrm{mM}$ EGTA, $10 \%$ glycerol) containing $10 \mu \mathrm{g} / \mathrm{ml} \mathrm{PMSF}$, complete protease inhibitor cocktail and $1 \%$ CHAPS. Cells were lysed for $30 \mathrm{~min}$ on ice followed by centrifugation for $10 \mathrm{~min}$ at $13,000 \mathrm{rpm}$ at $4^{\circ} \mathrm{C}$. The supernatant was diluted $1: 1$ with solubilization buffer without detergent and lysates were pre-cleared by incubation with Protein G sepharose beads (GE Healthcare) on a rotation wheel for $2 \mathrm{~h}$, at $4^{\circ} \mathrm{C}$. For immunoprecipitation, pre-cleared lysates were spun for $3 \min$ at $4,000 \times g$ at $4^{\circ} \mathrm{C}$, transferred to a clean tube and incubated with monoclonal anti-HA agarose beads (Sigma-Aldrich) for $4 \mathrm{~h}$, at $4^{\circ} \mathrm{C}$. Beads were washed three times with wash buffer (20 mM HEPES-KOH, pH 7.4, $150 \mathrm{mM} \mathrm{NaCl}, 1.5 \mathrm{mM} \mathrm{MgCl}_{2}$, $1 \mathrm{mM}$ EGTA, $10 \%$ glycerol) containing $0.1 \%$ CHAPS and eluted in 2x SDS-sample buffer (see below).

For determining the ubiquitination of endogenous SQS, Hek293T, Hek293T $\Delta$ SPP and Hek293T $\Delta$ TRC8 cells were seeded onto PLL-coated $100 \mathrm{~mm}$ cell culture dishes. Cells were washed two times with $1 \times$ PBS and treated overnight with medium containing $5 \%(v / v)$ LPDS. Prior to harvest, the proteasome inhibitor epoxomicin (final $5 \mu \mathrm{M}$ ) was added to each dish and one dish of each cell line received $30 \mu \mathrm{g} / \mathrm{ml} \mathrm{Chol} / \mathrm{CD}$ for 3 hours. Cells were washed once with 1x PBS and harvested in 1x PBS-EDTA supplemented with $20 \mathrm{mM} \mathrm{N}$ ethylmaleimide (NEM) to block deubiquitinating enzymes followed by centrifugation for 3 min at $900 \times g$ at $4^{\circ} \mathrm{C}$. Cells were lysed for $20 \mathrm{~min}$ on ice in $500 \mu$ l solubilization buffer containing freshly added protease inhibitor cocktail, PMSF, 1\% Triton X-100 and 20 mM NEM. Lysates were clarified by centrifugation for $15 \mathrm{~min}$ at full speed at $4^{\circ} \mathrm{C}$ and the supernatant was 
diluted 1:1 with solubilization buffer without detergent. Lysates were pre-cleared at $4^{\circ} \mathrm{C}$ for $1 \mathrm{~h}$ on a rotating wheel using $30 \mu \mathrm{l}$ Protein-S agarose beads (Merck Millipore) slurry. Beads were centrifuged for $3 \mathrm{~min}$ at $4000 \times \mathrm{g}$ and the pre-cleared lysate transferred to a fresh tube. Affinity purification/enrichment of ubiquitinated species was performed using $20 \mu \mathrm{I}$ TUBE2 agarose bead (LifeSensors) slurry, incubating overnight at $4^{\circ} \mathrm{C}$ on a rotating wheel. Beads were washed three times with TBS-T (50 mM Tris- $\mathrm{HCl}, \mathrm{pH} 7.4,150 \mathrm{mM} \mathrm{NaCl}, 0.1 \%$ Tween20). Proteins were eluted by heating to $65^{\circ} \mathrm{C}$ for $15 \mathrm{~min}$ in $20 \mu \mathrm{l} 1 \mathrm{x}$ SDS-sample buffer. Samples were subjected to SDS-PAGE and western blotting (see below).

\section{SDS-PAGE and western blotting}

Proteins were solubilized in SDS sample buffer (50 mM Tris-HCl, pH 6.8, 10 mM EDTA, pH 8.0, $5 \%$ glycerol, $2 \%$ SDS, $0.01 \%$ bromophenol blue) containing $5 \% \beta$-mercaptoethanol. Samples were heated to $65^{\circ} \mathrm{C}$ for 15 min with agitation, except for immunoblots of $\mathrm{Hrd} 1$ which were incubated at $37^{\circ} \mathrm{C}$ for $30 \mathrm{~min}$. For western blotting, proteins were separated by reducing Tris-glycine SDS-PAGE and transferred to polyvinylidene difluoride (PVDF, Merck Millipore) membranes followed by enhanced chemiluminescence analysis (Advansta) using the LAS4000 system (Fuji). For reprobing, PVDF membranes were incubated in glycine stripping buffer (100 mM glycine, $20 \mathrm{mM} \mathrm{Mg}(\mathrm{OAc})_{2}, 50 \mathrm{mM} \mathrm{KCl}, 1 \%$ Tween-20, 2.5\% SDS), $\mathrm{pH}$ 2.2, for $20 \mathrm{~min}$ followed by washing 6 times for $10 \mathrm{~min}$ with TBS-T before processing at outlined above.

\section{Statistical analysis}

Quantification of western blots was performed using ImageJ (http://rsb.info.nih.gov/ij/). Statistical analysis was performed using GraphPad Prism v.5.00 (GraphPad Software, Inc.). Differences between two means in steady state analysis was determined by two-tailed unpaired Student's $t$-test. The difference between multiple means in cycloheximide chase assays was determined by two-way ANOVA test followed by Bonferroni post hoc test. If not stated otherwise, data are represented as mean \pm SEM and result from at least three 
independent biological replicates. Significance levels were as follows: ${ }^{*} P \leq 0.05,{ }^{* *} P<0.01$, ${ }^{* * *} P<0.001$.

\section{Acknowledgments}

We thank Bernhard Dobberstein for reagents, Wilhelm Palm, Colin Adrain, Bernd Schröder and Torben Mentrup for advice and critical reading of the manuscript, Iris Leibrecht for technical support with the lipidome analysis and Thomas Ruppert (ZMBH MS facility) for MS/MS analysis. This work was supported by funds from the Deutsche Forschungsgemeinschaft LE 2749/2-1 to M.K.L., Project Number 112927078 - TRR 83 and Project Number 319506281 - TRR 186 to B.B.

\section{Author contribution}

R.H., D.A. and M.K.L. conceptualized the project. R.H., D.A. and M.K.L. designed the research and wrote the manuscript. R.H. and D.A. performed the experiments and analyzed the data. C.L., D.K., and B.B. performed the lipidome analysis and analyzed the data. H.B. and S.V. performed activity probe labelling experiments and analyzed the data. M.B.-X. synthesized activity probe. T.M. and B.S. performed experiments with mouse primary cells and analyzed the data.

\section{Declaration of Interests}

None declared. 


\section{References}

Aizawa S, Okamoto T, Sugiyama Y, Kouwaki T, Ito A, Suzuki T, Ono C, Fukuhara T, Yamamoto M, Okochi M, Hiraga N, Imamura M, Chayama K, Suzuki R, Shoji I, Moriishi K, Moriya K, Koike K, Matsuura Y (2016) TRC8-dependent degradation of hepatitis C virus immature core protein regulates viral propagation and pathogenesis. Nat Commun 7: 11379 Avci D, Lemberg MK (2015) Clipping or Extracting: Two Ways to Membrane Protein Degradation. Trends Cell Biol 25: 611-22

Avci D, Malchus NS, Heidasch R, Lorenz H, Richter K, Nessling M, Lemberg MK (2019) The intramembrane protease SPP impacts morphology of the endoplasmic reticulum by triggering degradation of morphogenic proteins. J Biol Chem 294: 2786-2800

Boname JM, Bloor S, Wandel MP, Nathan JA, Antrobus R, Dingwell KS, Thurston TL, Smith DL, Smith JC, Randow F, Lehner PJ (2014) Cleavage by signal peptide peptidase is required for the degradation of selected tail-anchored proteins. J Cell Biol 205: 847-62

Brauweiler A, Lorick KL, Lee JP, Tsai YC, Chan D, Weissman AM, Drabkin HA, Gemmill RM (2007) RING-dependent tumor suppression and G2/M arrest induced by the TRC8 hereditary kidney cancer gene. Oncogene 26: 2263-71

Brinkman EK, Chen T, Amendola M, van Steensel B (2014) Easy quantitative assessment of genome editing by sequence trace decomposition. Nucleic Acids Res 42: e168

Brown MS, Goldstein JL (1980) Multivalent feedback regulation of HMG CoA reductase, a control mechanism coordinating isoprenoid synthesis and cell growth. $J$ Lipid Res 21: 505-17

Brown MS, Radhakrishnan A, Goldstein JL (2018) Retrospective on Cholesterol Homeostasis: The Central Role of Scap. Annu Rev Biochem 87: 783-807

Cantagrel V, Lefeber DJ (2011) From glycosylation disorders to dolichol biosynthesis defects: a new class of metabolic diseases. J Inherit Metab Dis 34: 859-67

Chen C, Malchus NS, Hehn B, Stelzer W, Avci D, Langosch D, Lemberg MK (2014) Signal peptide peptidase functions in ERAD to cleave the unfolded protein response regulator XBP1u. EMBO J 33: 2492-506

Chun J, Yin YI, Yang G, Tarassishin L, Li YM (2004) Stereoselective synthesis of photoreactive peptidomimetic gamma-secretase inhibitors. J Org Chem 69: 7344-7

Coates HW, Capell-Hattam IM, Brown AJ (2021) The mammalian cholesterol synthesis enzyme squalene monooxygenase is proteasomally truncated to a constitutively active form. J Biol Chem: 100731 
Crump CJ, Ende CWA, Ballard TE, Pozdnyakov N, Pettersson M, Chau DM, Bales KR, Li YM, Johnson DS (2012) Development of clickable active site-directed photoaffinity probes for gamma-secretase. Bioorganic \& Medicinal Chemistry Letters 22: 2997-3000

Dehairs J, Talebi A, Cherifi Y, Swinnen JV (2016) CRISP-ID: decoding CRISPR mediated indels by Sanger sequencing. Scientific reports 6: 28973

Durocher Y, Perret S, Kamen A (2002) High-level and high-throughput recombinant protein production by transient transfection of suspension-growing human 293-EBNA1 cells. Nucleic Acids Res 30: E9

Edwards PA, Ericsson J (1999) Sterols and isoprenoids: signaling molecules derived from the cholesterol biosynthetic pathway. Annu Rev Biochem 68: 157-85

Foresti O, Ruggiano A, Hannibal-Bach HK, Ejsing CS, Carvalho P (2013) Sterol homeostasis requires regulated degradation of squalene monooxygenase by the ubiquitin ligase Doa10/Teb4. Elife 2: e00953

Gardner RG, Shearer AG, Hampton RY (2001) In vivo action of the HRD ubiquitin ligase complex: mechanisms of endoplasmic reticulum quality control and sterol regulation. Mol Cell Biol 21: 4276-91

Gill S, Stevenson J, Kristiana I, Brown AJ (2011) Cholesterol-dependent degradation of squalene monooxygenase, a control point in cholesterol synthesis beyond HMG-CoA reductase. Cell Metab 13: 260-73

Goldstein JL, Basu SK, Brown MS (1983) Receptor-mediated endocytosis of low-density lipoprotein in cultured cells. Methods Enzymol 98: 241-60

Goldstein JL, Brown MS (1990) Regulation of the mevalonate pathway. Nature 343: 425-30 Haeuptle MA, Hulsmeier AJ, Hennet T (2010) HPLC and mass spectrometry analysis of dolichol-phosphates at the cell culture scale. Anal Biochem 396: 133-8

Heigwer F, Kerr G, Boutros M (2014) E-CRISP: fast CRISPR target site identification. Nature methods 11: 122-3

Howe V, Sharpe LJ, Alexopoulos SJ, Kunze SV, Chua NK, Li D, Brown AJ (2016)

Cholesterol homeostasis: How do cells sense sterol excess? Chem Phys Lipids 199: 170178

Hsu FF, Chou YT, Chiang MT, Li FA, Yeh CT, Lee WH, Chau LY (2018) Signal peptide peptidase promotes tumor progression via facilitating FKBP8 degradation. Oncogene 
Hsu FF, Yeh CT, Sun YJ, Chiang MT, Lan WM, Li FA, Lee WH, Chau LY (2015) Signal peptide peptidase-mediated nuclear localization of heme oxygenase-1 promotes cancer cell proliferation and invasion independent of its enzymatic activity. Oncogene 34: 2360-70 Hua X, Sakai J, Brown MS, Goldstein JL (1996) Regulated cleavage of sterol regulatory element binding proteins requires sequences on both sides of the endoplasmic reticulum membrane. J Biol Chem 271: 10379-84.

Jiang LY, Jiang W, Tian N, Xiong YN, Liu J, Wei J, Wu KY, Luo J, Shi XJ, Song BL (2018) Ring finger protein 145 (RNF145) is a ubiquitin ligase for sterol-induced degradation of HMGCoA reductase. J Biol Chem 293: 4047-4055

Jo Y, Lee PC, Sguigna PV, DeBose-Boyd RA (2011) Sterol-induced degradation of HMG CoA reductase depends on interplay of two Insigs and two ubiquitin ligases, gp78 and Trc8. Proceedings of the National Academy of Sciences of the United States of America 108: 20503-8

Kaymak I, Maier CR, Schmitz W, Campbell AD, Dankworth B, Ade CP, Walz S, Paauwe M, Kalogirou C, Marouf H, Rosenfeldt MT, Gay DM, McGregor GH, Sansom OJ, Schulze A (2020) Mevalonate Pathway Provides Ubiquinone to Maintain Pyrimidine Synthesis and Survival in p53-Deficient Cancer Cells Exposed to Metabolic Stress. Cancer Res 80: 189-203 Klein U, Gimpl G, Fahrenholz F (1995) Alteration of the myometrial plasma membrane cholesterol content with beta-cyclodextrin modulates the binding affinity of the oxytocin receptor. Biochemistry 34: 13784-93

Lee JP, Brauweiler A, Rudolph M, Hooper JE, Drabkin HA, Gemmill RM (2010) The TRC8 ubiquitin ligase is sterol regulated and interacts with lipid and protein biosynthetic pathways. Mol Cancer Res 8: 93-106

Lemberg MK, Martoglio B (2002) Requirements for signal peptide peptidase-catalyzed intramembrane proteolysis. Mol Cell 10: 735-744

Lemberg MK, Strisovsky K (2021) Maintenance of organellar protein homeostasis by ERassociated degradation and related mechanisms. Molecular Cell: in press

Nadin A, Lopez JMS, Neduvelil JG, Thomas SR (2001) A stereocontrolled synthesis of 2Rbenzyl-5S-tert-butoxycarbonylamino-4R-(tert-butyldimethylsilanyloxy)-6-phenyl-hexanoic acid (Phe-Phe hydroxyethylene dipeptide isostere). Tetrahedron 57: 1861-1864

Nakanishi M, Goldstein JL, Brown MS (1988) Multivalent control of 3-hydroxy-3methylglutaryl coenzyme A reductase. Mevalonate-derived product inhibits translation of mRNA and accelerates degradation of enzyme. J Biol Chem 263: 8929-37 
Niemeyer J, Mentrup T, Heidasch R, Muller SA, Biswas U, Meyer R, Papadopoulou AA, Dederer V, Haug-Kroper M, Adamski V, Lullmann-Rauch R, Bergmann M, Mayerhofer A, Saftig P, Wennemuth G, Jessberger R, Fluhrer R, Lichtenthaler SF, Lemberg MK, Schroder B (2019) The intramembrane protease SPPL2c promotes male germ cell development by cleaving phospholamban. EMBO Rep 20

Ozbalci C, Sachsenheimer T, Brugger B (2013) Quantitative analysis of cellular lipids by nano-electrospray ionization mass spectrometry. Methods Mol Biol 1033: 3-20

Palm W, Araki J, King B, DeMatteo RG, Thompson CB (2017) Critical role for PI3-kinase in regulating the use of proteins as an amino acid source. Proceedings of the National Academy of Sciences of the United States of America 114: E8628-E8636

Paltauf F, Hermetter A (1994) Strategies for the synthesis of glycerophospholipids. Prog Lipid Res 33: 239-328

Pfaffl MW (2001) A new mathematical model for relative quantification in real-time RT-PCR. Nucleic Acids Res 29: e45

Rawson RB, Zelenski NG, Nijhawan D, Ye J, Sakai J, Hasan MT, Chang TY, Brown MS, Goldstein JL (1997) Complementation cloning of S2P, a gene encoding a putative metalloprotease required for intramembrane cleavage of SREBPs. Mol Cell 1: 47-57

Sandvig K, Torgersen ML, Raa HA, van Deurs B (2008) Clathrin-independent endocytosis: from nonexisting to an extreme degree of complexity. Histochem Cell Biol 129: 267-76 Schumacher MM, DeBose-Boyd RA (2021) Posttranslational Regulation of HMG CoA Reductase, the Rate-Limiting Enzyme in Synthesis of Cholesterol. Annu Rev Biochem 90: 659-679

Sever N, Song BL, Yabe D, Goldstein JL, Brown MS, DeBose-Boyd RA (2003) Insigdependent ubiquitination and degradation of mammalian 3-hydroxy-3-methylglutaryl-CoA reductase stimulated by sterols and geranylgeraniol. J Biol Chem 278: 52479-90

Skotland T, Sandvig K (2019) The role of PS 18:0/18:1 in membrane function. Nat Commun 10: 2752

Snapp EL, Sharma A, Lippincott-Schwartz J, Hegde RS (2006) Monitoring chaperone engagement of substrates in the endoplasmic reticulum of live cells. Proceedings of the National Academy of Sciences of the United States of America 103: 6536-41

Stagg HR, Thomas M, van den Boomen D, Wiertz EJ, Drabkin HA, Gemmill RM, Lehner PJ (2009) The TRC8 E3 ligase ubiquitinates MHC class I molecules before dislocation from the ER. J Cell Biol 186: 685-92 
Stefanovic-Barrett S, Dickson AS, Burr SP, Williamson JC, Lobb IT, van den Boomen DJ, Lehner PJ, Nathan JA (2018) MARCH6 and TRC8 facilitate the quality control of cytosolic and tail-anchored proteins. EMBO Rep 19

Ventura A, Kirsch DG, McLaughlin ME, Tuveson DA, Grimm J, Lintault L, Newman J, Reczek EE, Weissleder R, Jacks $T$ (2007) Restoration of p53 function leads to tumour regression in vivo. Nature 445: $661-5$

Wahrle S, Das P, Nyborg AC, McLendon C, Shoji M, Kawarabayashi T, Younkin LH, Younkin SG, Golde TE (2002) Cholesterol-dependent gamma-secretase activity in buoyant cholesterol-rich membrane microdomains. Neurobiol Dis 9: 11-23

Wei JW, Cai JQ, Fang C, Tan YL, Huang K, Yang C, Chen Q, Jiang CL, Kang CS (2017) Signal Peptide Peptidase, Encoded by HM13, Contributes to Tumor Progression by Affecting EGFRvIII Secretion Profiles in Glioblastoma. CNS Neurosci Ther 23: 257-265

Weihofen A, Binns K, Lemberg MK, Ashman K, Martoglio B (2002) Identification of signal peptide peptidase, a presenilin-type aspartic protease. Science 296: 2215-2218.

Yang G, Zhou R, Zhou Q, Guo X, Yan C, Ke M, Lei J, Shi Y (2019) Structural basis of Notch recognition by human gamma-secretase. Nature 565: 192-197

Yucel SS, Stelzer W, Lorenzoni A, Wozny M, Langosch D, Lemberg MK (2019) The Metastable XBP1u Transmembrane Domain Defines Determinants for Intramembrane Proteolysis by Signal Peptide Peptidase. Cell reports 26: 3087-3099 e11

Zelcer N, Sharpe LJ, Loregger A, Kristiana I, Cook EC, Phan L, Stevenson J, Brown AJ (2014) The E3 ubiquitin ligase MARCH6 degrades squalene monooxygenase and affects 3hydroxy-3-methyl-glutaryl coenzyme A reductase and the cholesterol synthesis pathway. Mol Cell Biol 34: 1262-70 


\section{Figure legends}

Figure 1. Differential organelle proteomics identifies SQS as an SPP substrate.

(A) Experimental outline of the SILAC-based organelle proteomics.

(B) ER-resident membrane proteins quantified by organelle proteomics (mean $\mathrm{H} / \mathrm{L}$ ratio, $n=2)$.

(C) Western blot (WB) showing steady state analysis of endogenous SQS and HMGCR in Hek293T cells upon $16 \mathrm{~h}$ treatment with (Z-LL) $)_{2}$-ketone or DMSO. Actin was used as loading control. Quantification shows Mean \pm SEM, $n=4$.

(D) Cycloheximide (CHX) chase of endogenous SQS in Hek293T cells in presence of (Z$\mathrm{LL})_{2}$-ketone or vehicle control (DMSO). Monomeric and SDS-stable dimeric forms of SPP are indicated ( $m$ and $d$, respectively). Actin was used as loading control. Mean \pm SEM, $n=3$.

(E) CHX chase of ectopically expressed FLAG-tagged SQS in Hek293T cells co-expressing HA-tagged wild-type (wt) SPP and catalytic mutant SPP $^{\mathrm{D} 265 \mathrm{~A}}$ (DA) revealing processing of the full-length form (black arrow) to an $\mathrm{N}$-terminal fragment (white arrow).

(F) Western blot analysis showing increased SQS levels upon tamoxifen induced SPP knock out in mice bone marrow-derived dendritic cells. Western blot and quantification show 4 biological replicates. Actin was used as loading control.

Figure 2. SPP and TRC8 define a non-canonical ERAD pathway that acts on SQS in parallel to Hrd1 pathway.

(A) Stability of endogenous SQS in Hek293T wt and Hek293T $\triangle$ SPP cells assessed by cycloheximide $(\mathrm{CHX})$ chase assay. Actin was used as loading control. Mean $\pm \mathrm{SEM}, n=3$.

(B) SQS degradation assay in Hek293T wt, $\triangle T R C 8, \triangle M A R C H 6$, and $\triangle T R C 8 \triangle M A R C H 6$ $(\Delta \mathrm{T} \Delta \mathrm{M})$ double-deficient Hek293T cells. Mean $\pm \mathrm{SEM}, n=3$.

(C) SQS degradation assay in $\triangle \mathrm{T} \Delta \mathrm{M}$ cells transfected with non-targeting (nt) or Hrd1 siRNA. Mean \pm SEM, $n=3$.

Figure 3. Cholesterol activates SPP-TRC8 complex for regulatory degradation of SQS. 
(A) Experimental outline of the sterol starvation and cholesterol repletion assay. LPDS, lipoprotein-depleted serum; Chol/CD, cholesterol complexed to methyl- $\beta$-cyclodextrin.

(B) Degradation of endogenous SQS and the full-length form of SQLE (black arrow) were assayed in Hek293T wt cells treated as outlined in (A) and analyzed by western blot (WB) analysis. Actin was used as loading control. Grey arrow, cholesterol-insensitive truncated SQLE-S. Mean \pm SEM, $n=3$.

(C) SQS and SQLE degradation assay in Hek293T $\triangle$ SPP cells as in (B).

(D) SQS and SQLE degradation assay in Hek293T $\triangle T R C 8$ cells as in (B).

(E) Hek293T wt, $\triangle$ SPP, and $\triangle T R C 8$ cells were grown in sterol-depletion medium and treated with epoxomicin and incubated in cholesterol depletion or repletion as outlined in (A) before cell lysates were subjected to affinity enrichment using TUBE2 agarose beads revealing ubiquitinated species (white arrows). Control (Ctrl.) agarose beads were used to confirm TUBE2 specificity.

Figure 4. TRC8 acts as an allosteric activator of SPP-catalyzed SQS cleavage.

(A) Outline of TRC8 constructs used.

(B) Doxycycline-induced expression of HA-tagged TRC8 wt or the Y32E mutant in Hek293ATRC8 cells. Cells were subjected to CHX chase and assayed for endogenous SQS. Mean \pm SEM, $n=3$.

(C) Doxycycline-induced expression of HA-tagged TRC8 wt or RING domain mutant (RING ${ }^{\text {mt }}$ ) in Hek293 $\Delta$ TRC8 cells. Cells were subjected to CHX chase and assayed for endogenous SQS. Mean \pm SEM, $n=3$.

(D) Doxycycline-induced expression of HA-tagged TRC8 wt or the RING ${ }^{\mathrm{mt}}$ in Hek293 $\mathrm{TRC}_{\mathrm{T}}$ cells in cholesterol depletion or repletion. Cells were assayed for endogenous SQS by CHX chase as outlined in (Fig 3A). Mean \pm SEM, $n=3$.

(E) Doxycycline-induced expression of HA-tagged TRC8 RING ${ }^{m t}$ or the RING ${ }^{\text {mt }}$ Y32E double mutant in Hek293 $\triangle$ TRC8 cells. Cells were subjected to $\mathrm{CHX}$ chase assay and assayed for endogenous SQS. Mean \pm SEM, $n=3$. 
(F) Doxycycline-induced expression of HA-tagged TRC8-RING ${ }^{\mathrm{mt}}$ in Hek293 $\Delta$ TRC8 cells was assayed for endogenous SQS by $\mathrm{CHX}$ chase in presence or absence of $(Z-L L)_{2}$-ketone (inh.). Mean \pm SEM, $n=4$.

(G) Activity probe labelling of endogenous SPP-S-tag in microsomes isolated from cells grown in FBS or LPDS containing medium. SDS-PAGE analysis and quantification of probe binding is shown for 4 independent experiments.

\section{Figure 5. SPP-mediated SQS quantity regulation plays a crucial role in the control of} cholesterol homeostasis.

(A) Lipidomic analysis of subcellular fractionation samples comparing Hek293T wt and three $\Delta$ SPP cell lines (clone \#1, \#2, \#3). Mean \pm SEM, $n=3$.

(B) Hek293T wt and $\triangle$ SPP cells were grown in sterol-depletion medium (top panel) or in cholesterol-rich medium (bottom panel) and incubated with fluorescently labelled $70-k \mathrm{Da}$ dextran (green) and 10-kDa dextran (red). Intracellular dextran was quantified after 30 min uptake. Nuclear DNA was stained by Hoechst 33342 (blue). Mean \pm SEM, $n=10$ fields of view. Scale bar, $100 \mu \mathrm{m}$.

(C) Model for SPP-TRC8-mediated switch between the non-sterol and sterol branches of the mevalonate pathway.

(D) Lipidomic analysis of DolP levels comparing Hek293T wt and three $\Delta$ SPP cell lines (clone \#1, \#2, \#3). Quantification is shown for 4 replicates.

Figure 6. Model for SPP-triggered cholesterol dependent degradation of SQS.

At high level, cholesterol is sensed by the sterol-sensing domain (SSD) of TRC8 in Hek293T wt cells leading to ubiquitination and subsequent SPP-mediated ERAD-R of SQS. 


\section{Expanded View Figure legends}

Figure EV1. TM region of SQS targets it for SPP-mediated ERAD, related to Fig 1.

(A) Simplified view of the branched mevalonate pathway leading to cholesterol biosynthesis by multiple steps (black arrows) and other isoprenoids including dolichol, which in its phosphorylated form (dolichol-P) serves as the lipid carrier for glycans in the ER (Cantagrel \& Lefeber, 2011), ubiquinone and heme (blue arrows) (Goldstein \& Brown, 1990).

Biosynthesis starts from acetyl-CoA, which derives from glucose metabolism and the breakdown of amino acids and fatty acids. The conversion of HMG-CoA to mevalonate by HMGCR is considered as the rate-limiting step. The intermediate FPP (farnesylpyrophosphate) can be shunted either into the non-sterol branch or the sterol-branch ultimately leading to cholesterol. The conversion of FPP to squalene by SQS is the first committed step in the sterol-branch. The over-all rate-limiting enzyme HMGCR is posttranslationally regulated by ERAD involving multiple E3 ubiquitin ligases (red box) (Gardner et al., 2001, Jiang et al., 2018, Jo et al., 2011, Zelcer et al., 2014); SQLE and the yeast orthologue Erg1 are degraded by MARCH6/Doa10 (Foresti et al., 2013, Gill et al., 2011, Zelcer et al., 2014).

(B) Sub-cellular fractionation of Hek293T cells ectopically expressing SPP reveals that, after $\mathrm{CHX}$ chase for $8 \mathrm{~h}$ in presence of proteasome inhibitor epoxomicin (epox.) an SQS species is detected in the cytoplasmic fraction using the SQS antibody; ab195046. Addition of the SPP inhibitor $(Z-L L)_{2}-$ ketone blocks generation of this fragment. $t$, total cell extract, $m$, membrane fraction, s: supernatant.

(C) $\mathrm{CHX}$ chase of co-expressed FLAG-tagged Ubc6 with HA-tagged wt or DA-mutant SPP. Degradation of Ubc6 is not affected by SPP. Actin was used as loading control.

(D) Sequence alignment of human (Homo sapiens, Hs), rat (Rattus norvegicus, Rn), mouse (Mus musculus, Mm), and zebrafish (Danio rerio, Dr) SQS homologs shows conserved serine residues (bold) in the TM domain.

(E) Immunofluorescence microscopy of transiently transfected Hek293T cells with FLAGtagged SQS constructs as indicated (green) co-expressing the luminal ER marker RFP- 
KDEL (red) showing ER localization. Five frames, each from Z-stacks with step sizes of $0.5 \mu \mathrm{m}$, are displayed. Hoechst 33342, nuclear counterstaining, is shown in blue. Scale bar, $10 \mu \mathrm{m}$.

(F) Stability of ectopically expressed FLAG-tagged SQS wild-type (wt) and the TM domain mutants S388L and S397L was analyzed by $\mathrm{CHX}$ chase. Mean \pm SEM, $n=3$.

(G) Co-immunoprecipitation assay with ectopically expressed HA-tagged SPP wt or DA mutant and endogenous SQS. Asterisks represent antibody heavy and light chain.

Figure EV2. SQS is degraded by a consorted action of SPP and TRC8, related to Fig 2.

(A) Functional validation Hek293T $\triangle$ SPP cells by analysis of steady state levels of endogenous HO1. Hek293T wt and two Hek293T $\Delta$ SPP clones (\#1, \#2) were overnight treated with $20 \mu \mathrm{M}$ hemin in order to induce expression of endogenous $\mathrm{HO} 1$ and subjected to immunoblotting. Endogenous $\mathrm{HO} 1$ is significantly accumulating in both $\triangle \mathrm{SPP}$ clones. Immunoblot for SPP shows loss of protein. Only the monomeric form of SPP in Hek293T wt cells is shown. Mean \pm SEM; $n=3$.

(B) Functional validation of $\triangle \mathrm{SPP}$ cells in respect to processing of the hepatitis $\mathrm{C}$ virus $(\mathrm{HCV})$ nucleocapsid (core) protein. Hek293T wt and two Hek293T $\Delta$ SPP clones (\#1, \#2) were transiently transfected with an N-terminally FLAG-tagged construct comprising the HCV core protein $(\mathrm{C})$ followed by 4 amino acids of the $\mathrm{E} 1$ glycoprotein $(\mathrm{E} 1 / 4)$ as has been used before (Lemberg \& Martoglio, 2002). Cells were treated overnight in absence or presence of the proteasome inhibitor epoxomicin and subjected to immunoblotting. FLAG-tagged CE1/4 is first processed to the 191-residue immature core protein $(\mathrm{C} / 191)$ by signal peptidase and then to 179 amino acid long mature core protein (C/179) by SPP. Cleavage of $C / 191$ is abolished in $\triangle$ SPP cells leading to its degradation by ERAD as has been shown (Aizawa et al., 2016). Immunoblot for SPP shows loss of protein in both $\triangle$ SPP clones and the monomeric form of SPP in Hek293T wt cells is indicated. GFP was used as transfection control and actin was used as loading control. 
(C) Western blot (WB) showing the significant accumulation of endogenous SQS in two different Hek293T $\triangle$ SPP clones (\#1, \#2) whereas HMGCR was unaffected. Only the monomeric from of SPP is shown. Actin was used as loading control. Mean \pm SEM, $n=3$. (D) Quantitative real-time (qRT)-PCR for SQS (transcribed from the FDFT1 gene) was performed on mRNA isolated from Hek293T wt and two Hek293T $\triangle$ SPP clones (\#1, \#2). Level of SQS mRNA were normalized to TBP and $\beta 2 \mathrm{M}$ and is shown as fold-change relative to wt mRNA level. Technical triplicates from three independent biological replicates were performed. Mean \pm SEM, $n=3$.

(E) qRT-PCR for SQS/FDFT1 as in (D) but in two $\triangle T R C 8$ clones $(\Delta T \# 1, \Delta T \# 2)$, two $\Delta \mathrm{MARCH} 6$ clones $(\Delta \mathrm{M} \# 1, \Delta \mathrm{M} \# 2)$, and one $\triangle \mathrm{TRC} 8 \Delta \mathrm{MARCH} 6(\Delta \mathrm{T} \Delta \mathrm{M})$ double-knockout. Mean \pm SEM, $n=3$.

(F) CHX chase of endogenous SQLE and SQS in Hek293T wt and $\triangle M A R C H 6$ cells showing striking stabilization of SQLE, but not of SQS. Black arrow; full length SQLE, grey arrow; SQLE-S.

(G) CHX chase of endogenous SQS for indicated time-points in Hek293T wt cells transfected with non-targeting (nt) or Hrd1-specific siRNA. Mean \pm SEM, $n=3$.

(H-J) CHX chase of endogenous SQS in $\triangle \mathrm{SPP}(\mathbf{H}), \triangle \mathrm{TRC} 8(\mathbf{I})$, and $\triangle \mathrm{MARCH6}(\mathbf{J})$ Hek293T cells in presence of $(Z-L L)_{2}$-ketone or DMSO (vehicle). Mean \pm SEM, $n=3$.

Figure EV3. Cholesterol but not 25-hydroxycholesterol triggers SPP-TRC8-dependent ERAD of SQS and HO1, related to Fig 3.

(A) Crystal violet cytotoxicity assay shows that cell viability was not affected by the cholesterol depletion protocol outlined in Fig 3A. Hek293T wt, $\triangle$ SPP, or $\triangle T R C 8$ cells were seeded in 24-well plates, treated as indicated and assayed for cytotoxicity by crystal violet staining.

(B) Western blot analysis showing effect of cholesterol and 25-hydroxycholesterol (25-HC) on the degradation of endogenous HMGCR, SQS and SQLE. Hek293T wt were grown in medium containing FBS or LPDS overnight and sterol depleted cells were treated for $5 \mathrm{~h}$ with 
either $30 \mu \mathrm{g} / \mathrm{ml} \mathrm{Chol} / \mathrm{CD}$ or $1 \mu \mathrm{g} / \mathrm{ml} 25-\mathrm{HC}$, as indicated. Western blot analysis of two independent experiments are shown. Black arrow; full length SQLE, grey arrow; SQLE-S. (C and D) Turnover of endogenous HO1 by cholesterol was assayed in Hek293T wt (C) and Hek293T $\Delta$ SPP (D) cells. HO1 induction with $20 \mu \mathrm{M}$ hemin and sterol depletion was performed overnight. Cells were subjected to $\mathrm{CHX}$ chase for indicated time points in either absence or presence of $30 \mu \mathrm{g} / \mathrm{ml} \mathrm{Chol} / \mathrm{CD}$ and immunoblotted for endogenous HO1. SQLE was used as positive control for the assay. Asterisk, unspecific band. Actin was used as loading control. Mean \pm SEM, $n=3$.

Figure EV4. HB-169 specifically labels SPP. (A) The peptide backbone of HB-169 was partially constructed by solid phase synthesis, from the precursor 1 the peptide chain was elongated via standard Fmoc chemistry by consecutive deprotection and subsequent amino acid coupling as described in the Materials and Methods. The amino acids used are Ahx, Ahx, Bpa and Leu, represented by $\mathbf{i}$ in the synthetic scheme. (B) Activity probe labelling of SPP in microsomes from endogenously S-tagged cells, in presence or absence of $(Z L L)_{2^{-}}$ ketone as competing inhibitor for HB-169. Asterisk, TAMRA signal is specific for SPP and is lost upon addition of $(Z L L)_{2}$-ketone.

Figure EV5. Lipidomic profile of $\Delta$ SPP cells, related to Fig 5.

(A) Lipid class profile of Hek293T wt and three Hek293T $\triangle$ SPP $(\# 1, \# 2, \# 3)$ cell lines. Lipid classes are standardized to all lipids measured and lipid categories are indicated. PC, phosphatidylcholine; PE, phosphatidylethanolamine; PS, phosphatidylserine; PI, phosphatidylinositol; PG, phosphatidylglycerol; PA, phosphatidic acid; Cer, ceramide; SM, sphingomyelin; HexCer, hexosylceramide; CE, cholesteryl ester; DAG, diacylglycerol; TAG, triacylglycerol. Mean \pm SEM, $n=4$.

(B-E) Molecular species profiles of PE (B), PS (C), PC (D) and PC O (E) are shown as \% distributions within each class. Mean \pm SEM; $n=4$. 
(F) Lipidomic analysis of total cholesterol levels in Hek293T wt and three independent $\Delta$ SPP clones $(\# 1, \# 2, \# 3)$. Mean \pm SEM, $n=4$.

(G) Lipidomic analysis of functional lipid categories compared in Hek293T wt and three $\Delta$ SPP clones (\#1, \#2, \#3). GPL, glycerophospholipids; SP, sphingolipids. Storage lipids include cholesteryl ester, diacylglycerol and triacylglycerol. Mean \pm SEM, $n=4$.

Table EV1, related to Fig 1. Complete list of proteins identified in high-salt washed and carbonate-extracted microsomes by SILAC-based quantitative proteomics. Lists for all ERresident and all identified TA-proteins are given. Proteins enriched $>20 \%$ are highlighted in blue; protein depleted $>20 \%$ are highlighted yellow. 
Heidasch et al., Figure 1

A

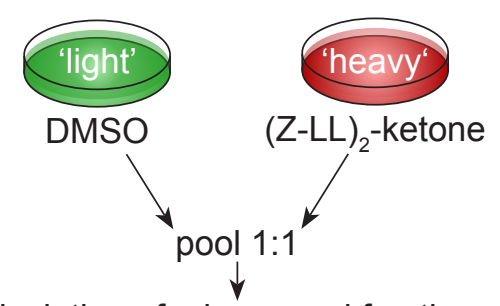

isolation of microsomal fraction

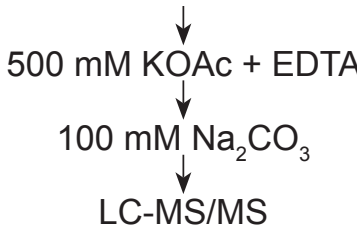

B

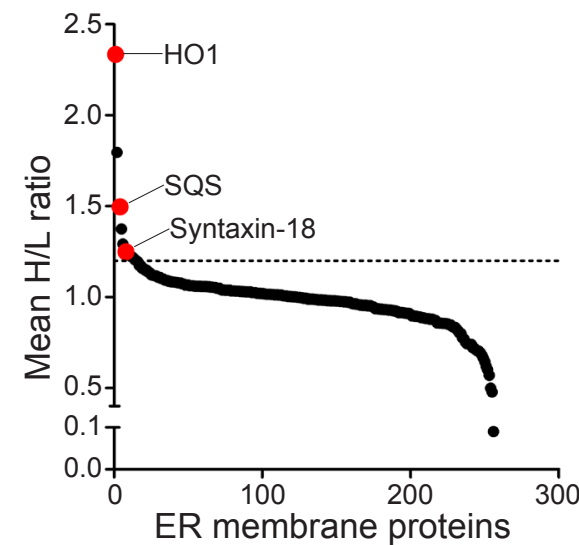

C

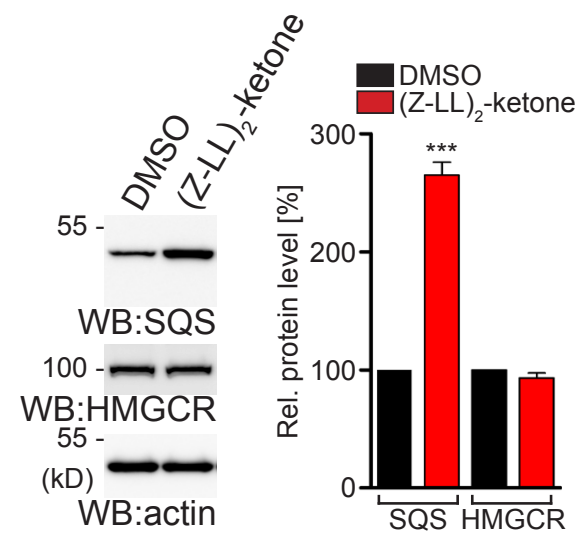

D

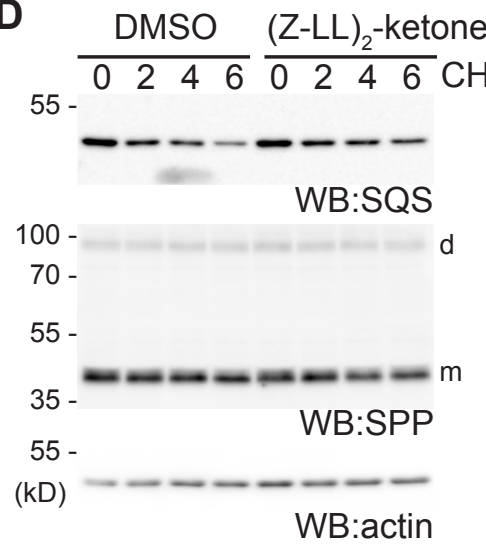

$\mathbf{F}$

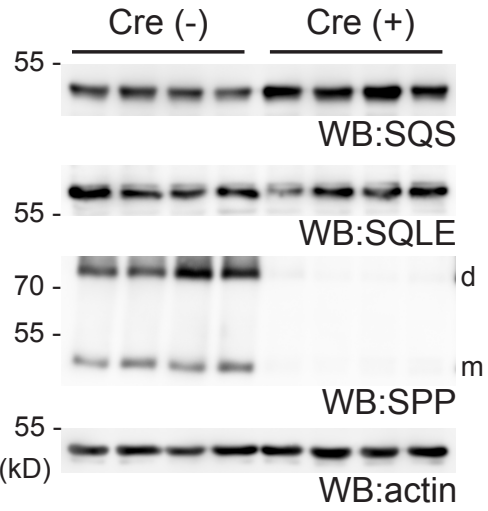

E
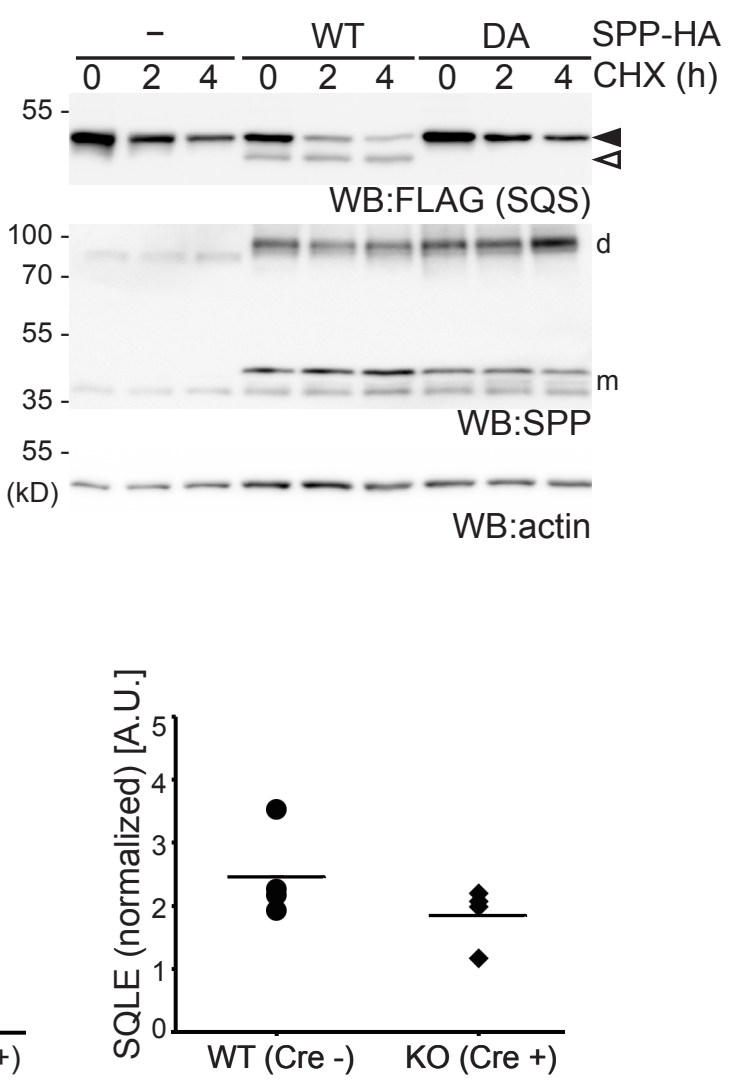
Heidasch et al., Figure 2

A
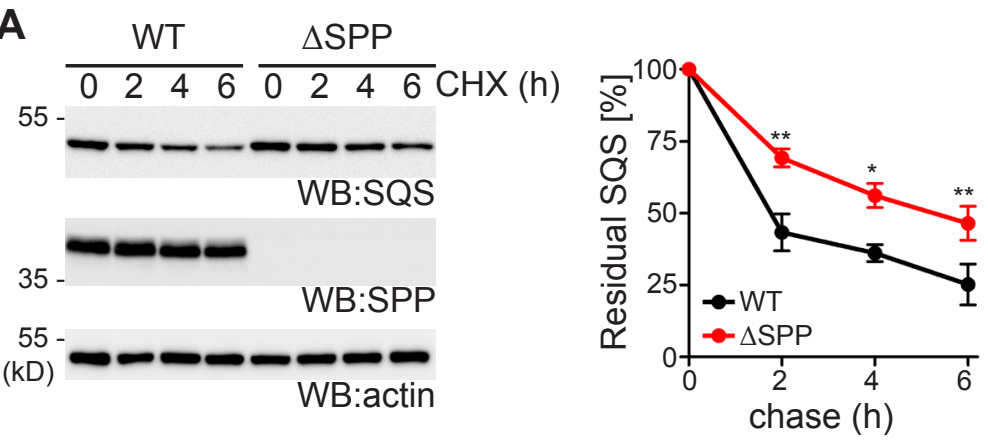

B
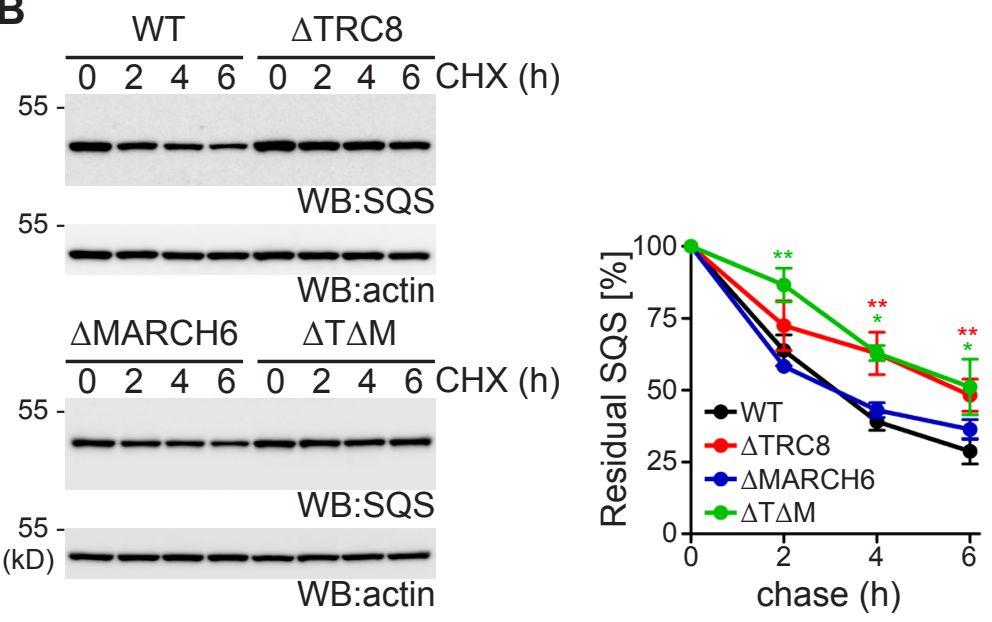

C
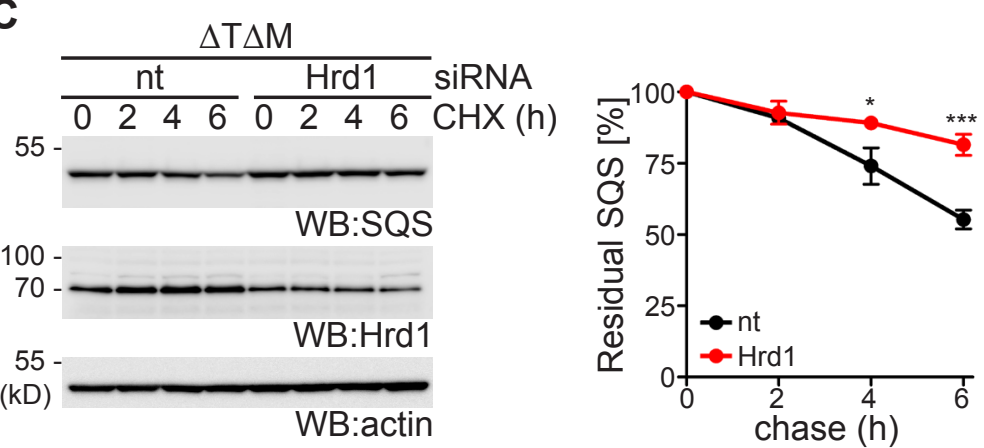
Heidasch et al., Figure 3

A

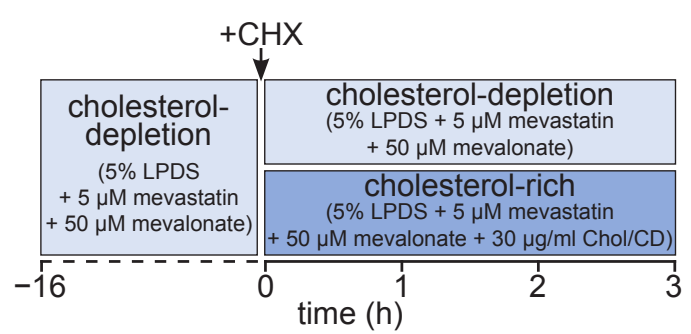

C

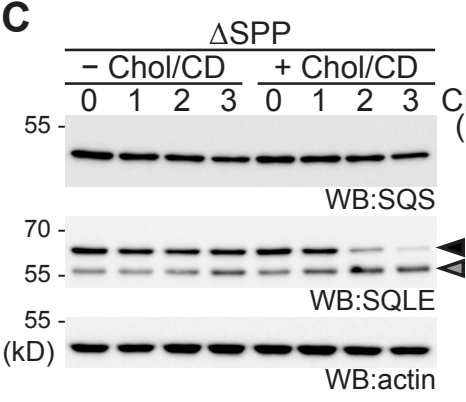

E

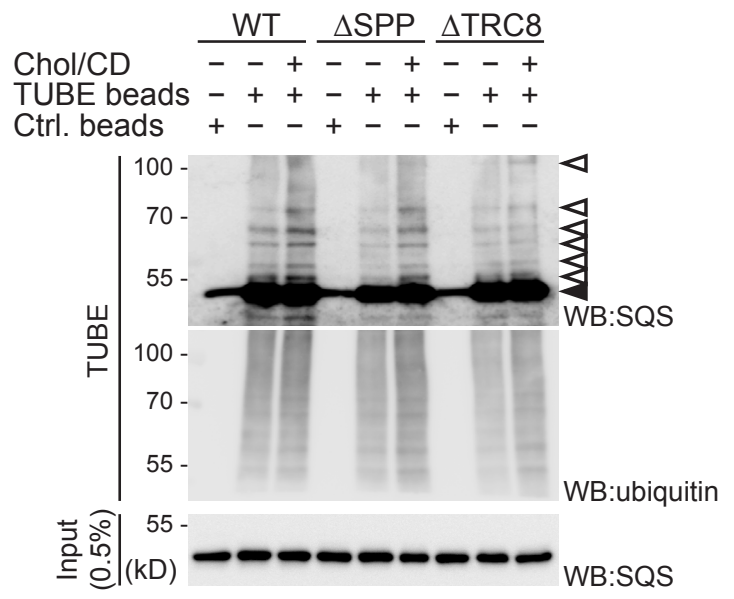

B

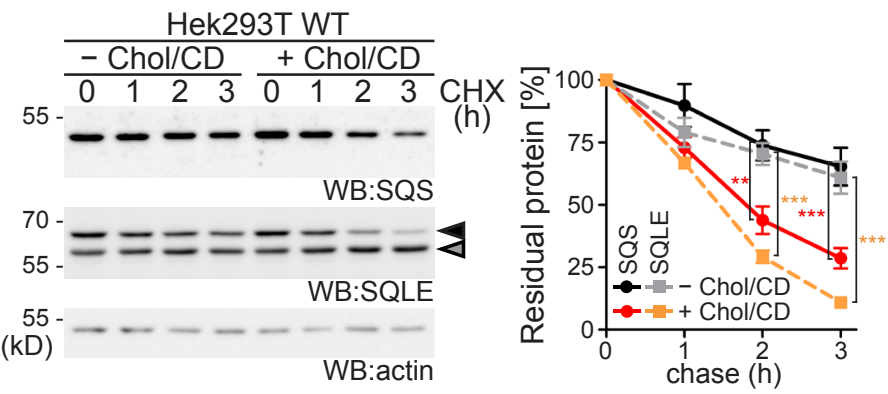

D
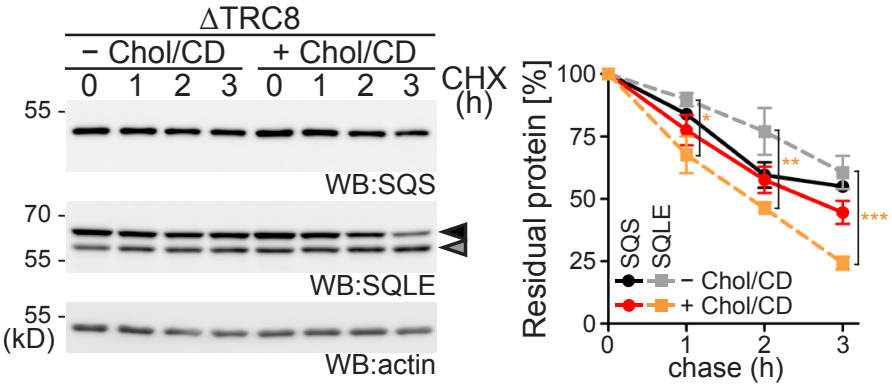
Heidasch et al., Figure 4

A

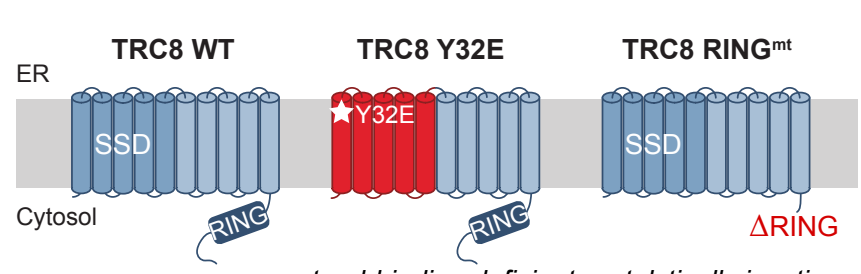

sterol-binding deficient catalytically inactive
C

$\frac{\Delta \text { TRC8 }}{\frac{-}{024} \frac{\text { WT }}{024} \frac{\text { RING }^{m i t}}{024}}$ CHX (h)

WB:SQS

$70-$

$55-$

(kD)

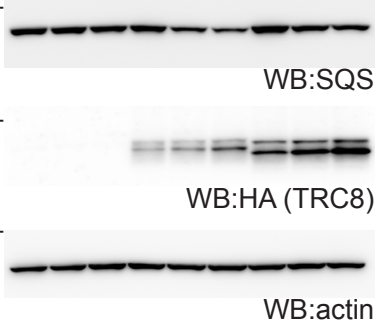

E

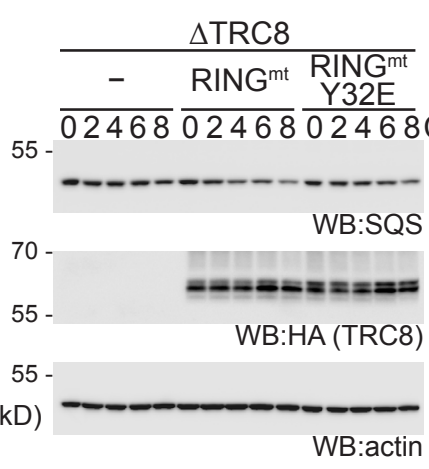

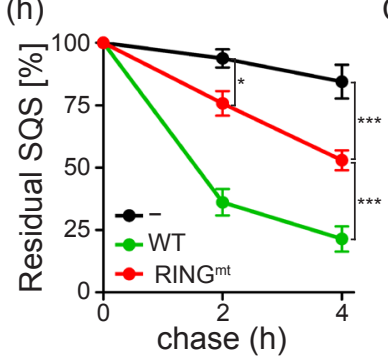

D

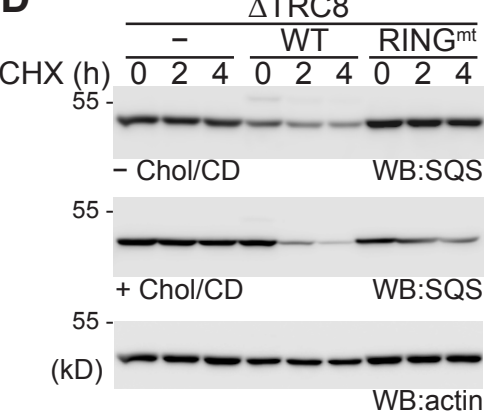

(h)

WB:SQS

WB:HA (TRC8)

$(\mathrm{KD})$

WB:actin

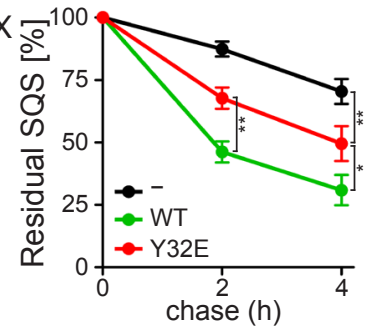

- $\triangle \mathrm{TRC} 8,-\mathrm{Chol} / \mathrm{CD}$

- $\triangle \mathrm{TRC} 8,+\mathrm{Chol} / \mathrm{CD}$

- $\triangle \mathrm{TRC} 8,+\mathrm{wt},-\mathrm{Chol} / \mathrm{CD}$

ㅎ $\triangle \mathrm{TRC} 8,+\mathrm{wt},+\mathrm{Chol} / \mathrm{CD}]^{*}$

- $\triangle$ TRC8, + RINGmt,$-\mathrm{Chol}_{\text {/CD }}$

$\left.\triangle \Delta \mathrm{TRC},+\mathrm{RING}{ }^{\mathrm{mt}},+\mathrm{Chol} / \mathrm{CD}\right]^{* * *}$

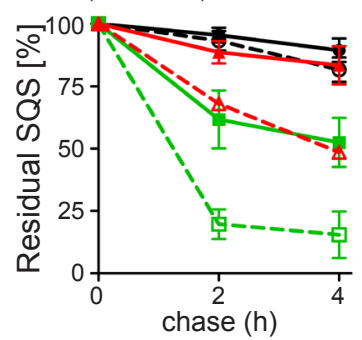

$\mathbf{F}$

F $\Delta$ TRC8+TRC8-RING ${ }^{\mathrm{mt}}$
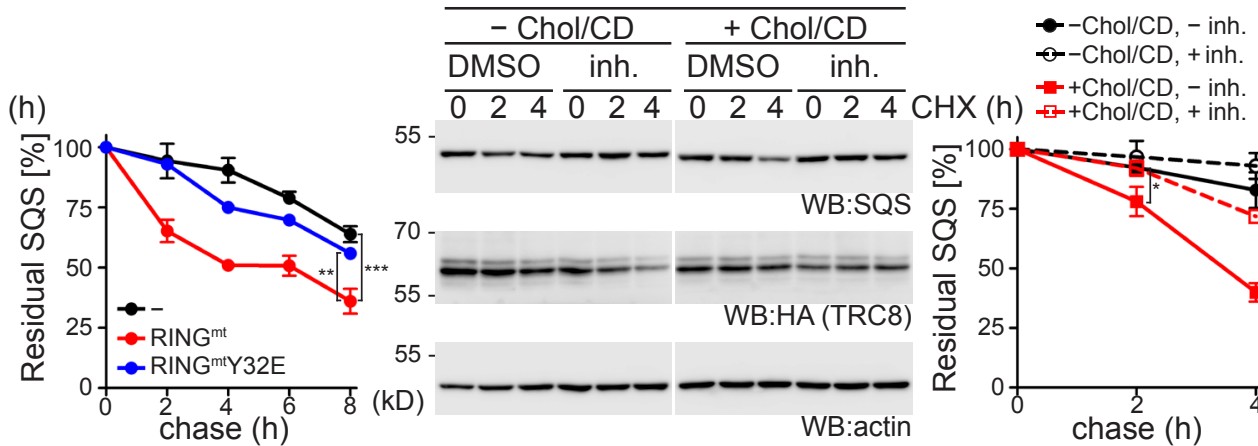
55
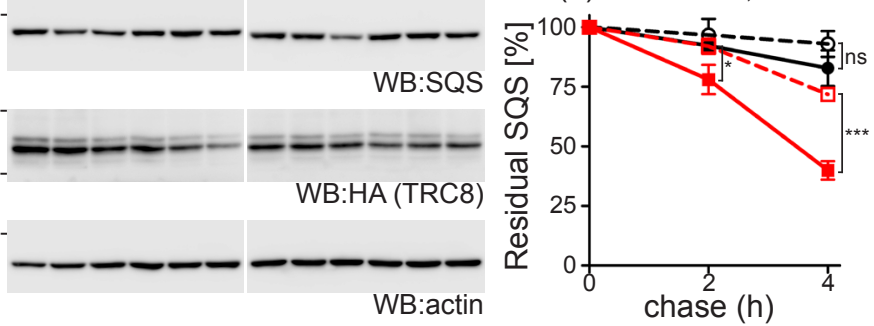

G
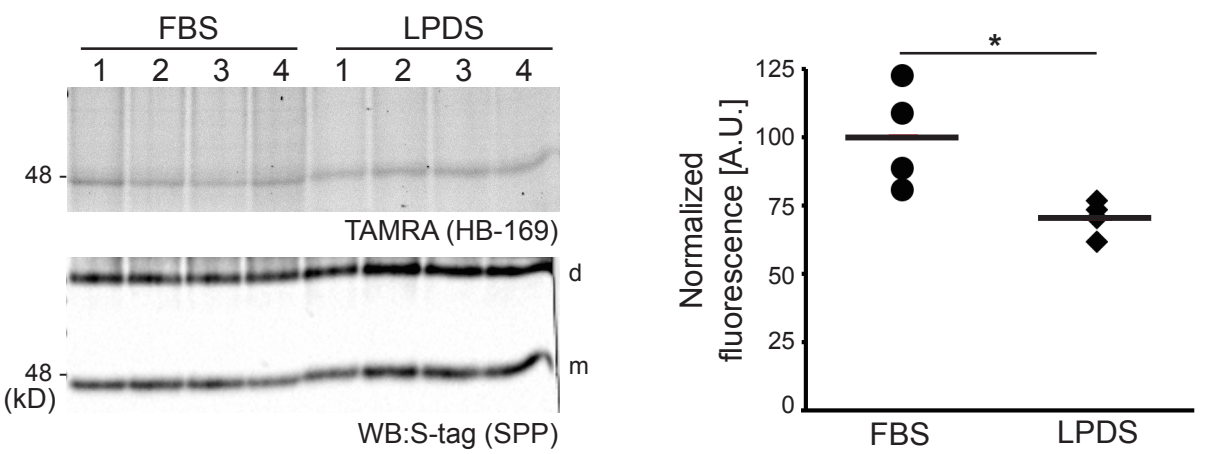
Heidasch et al., Figure 5

A

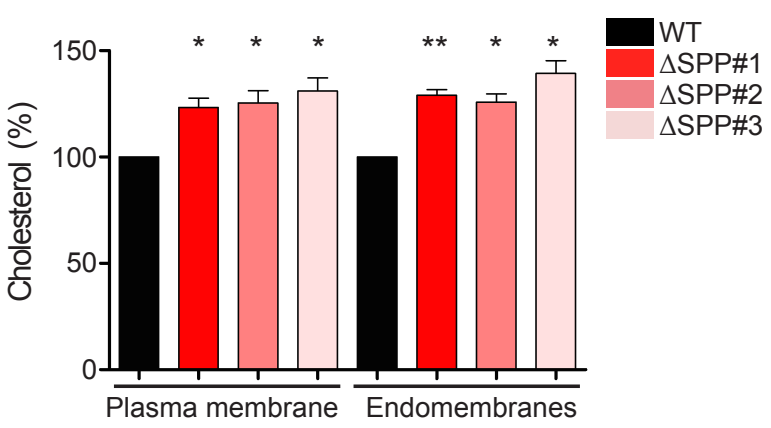

C

mevalonate pathway

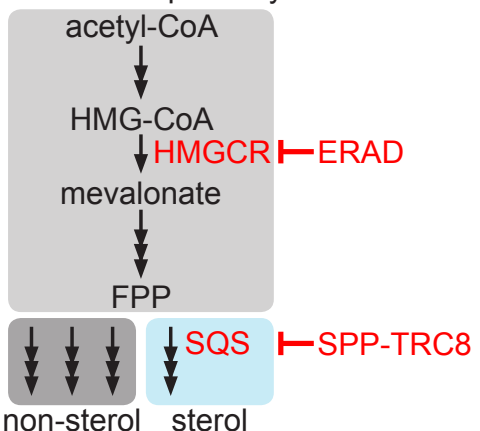

D

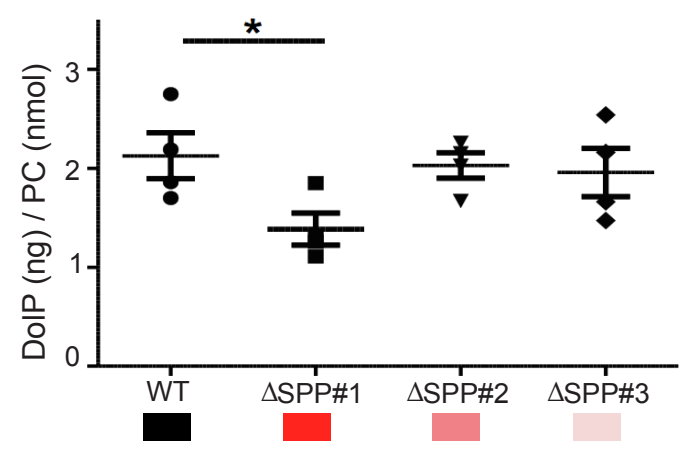

B
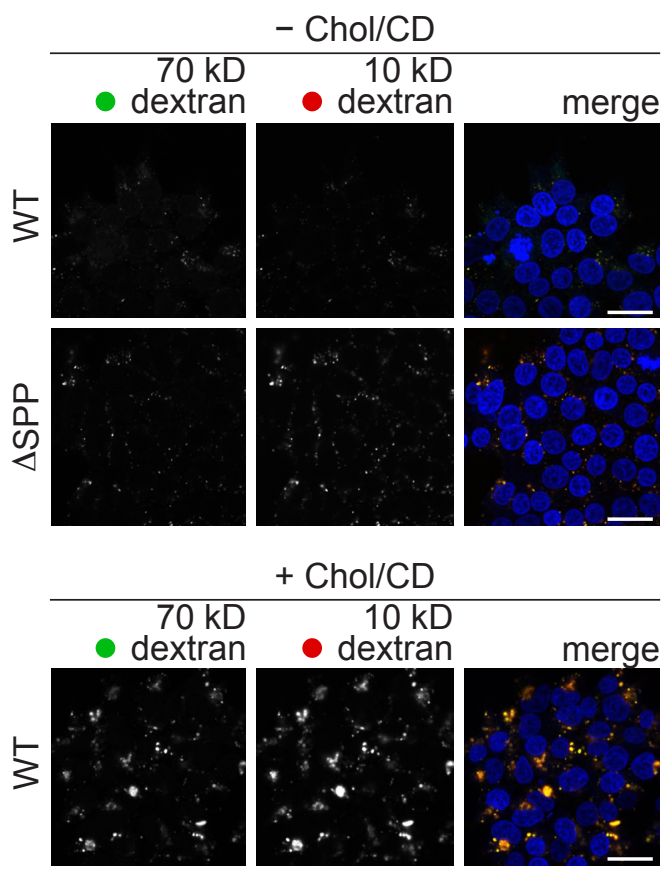

$+\mathrm{Chol} / \mathrm{CD}$

$10 \mathrm{kD}$

- dextran
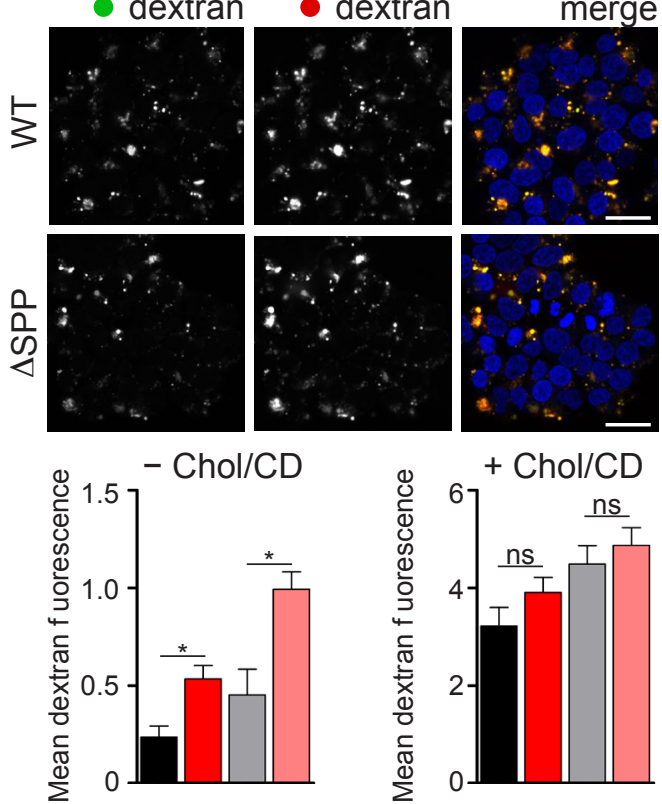

WT $\triangle$ SPP

$70 \mathrm{kD}$ dextran $10 \mathrm{kD}$ dextran 
bioRxiv preprint doi: https://doi.org/10.1101/2021.07.19.452877; this version posted July 20, 2021. The copyright holder for this preprint (which was not certified by peer review) is the author/funder. All rights reserved. No reuse allowed without permission.

Heidasch et al., Figure 6

high cholesterol

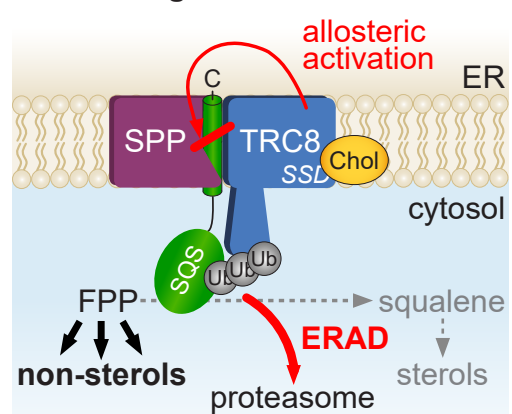

